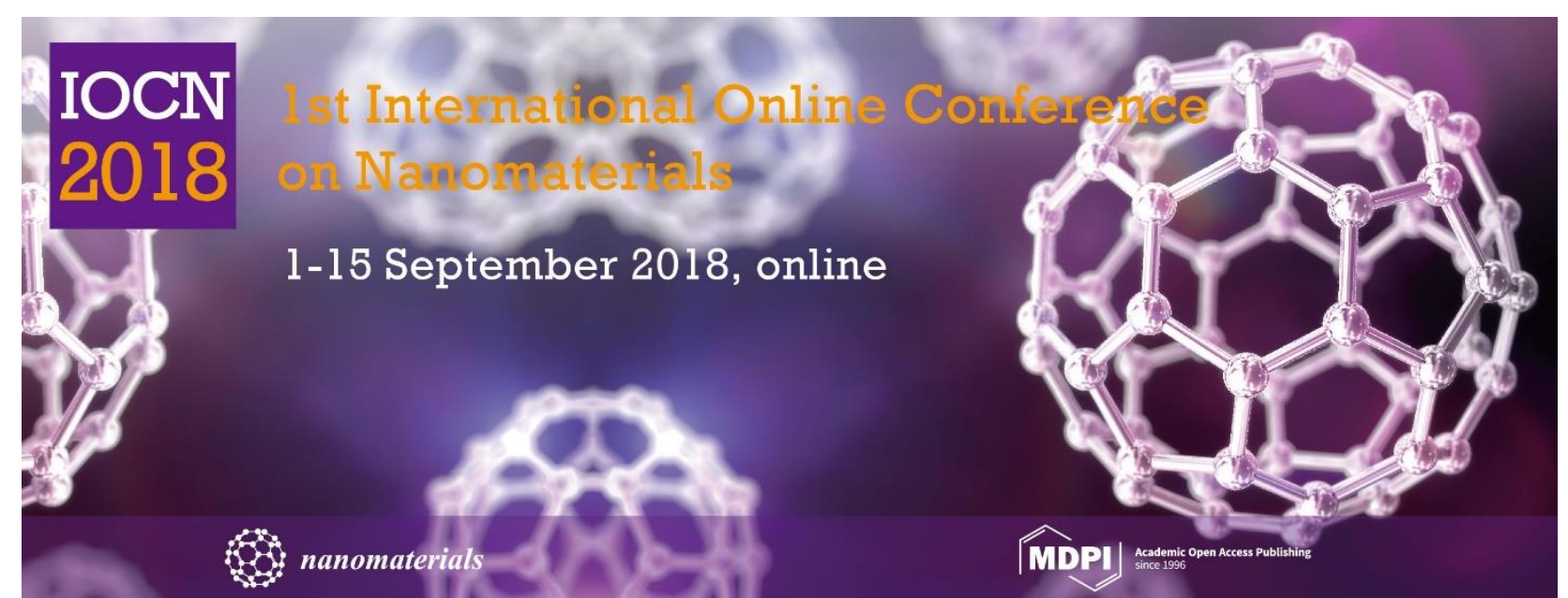

\title{
Preclinical development of a molecularly-defined liposomal vaccine for cutaneous leishmaniasis
}

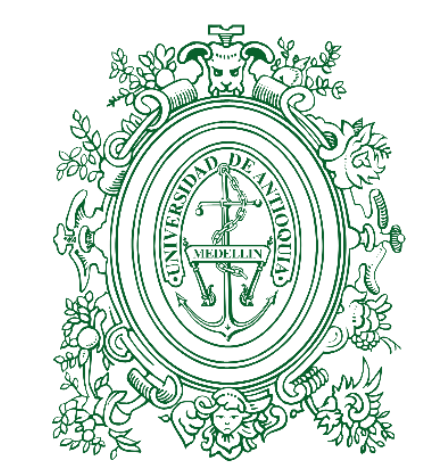

UNIVERSIDAD DE ANTIOQUIA
David Ernesto Bautista-Erazo ${ }^{1}$ Natalia García-Valencia ${ }^{1}$

Verónica Guzmán-González ${ }^{1}$ Gisela María García-Montoya² José Robinson Ramírez-Pineda ${ }^{1 *}$

*Correspondence: ramirezpineda@yahoo.com

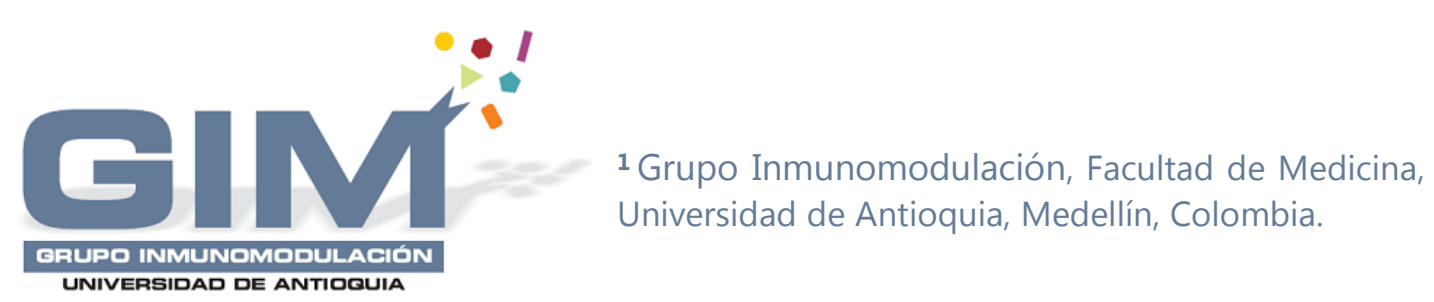

CENTRO NACIONAL DE SECUENCIACIÓ GENONICA - CNSG 


\section{Leishmaniasis: a public health problem}
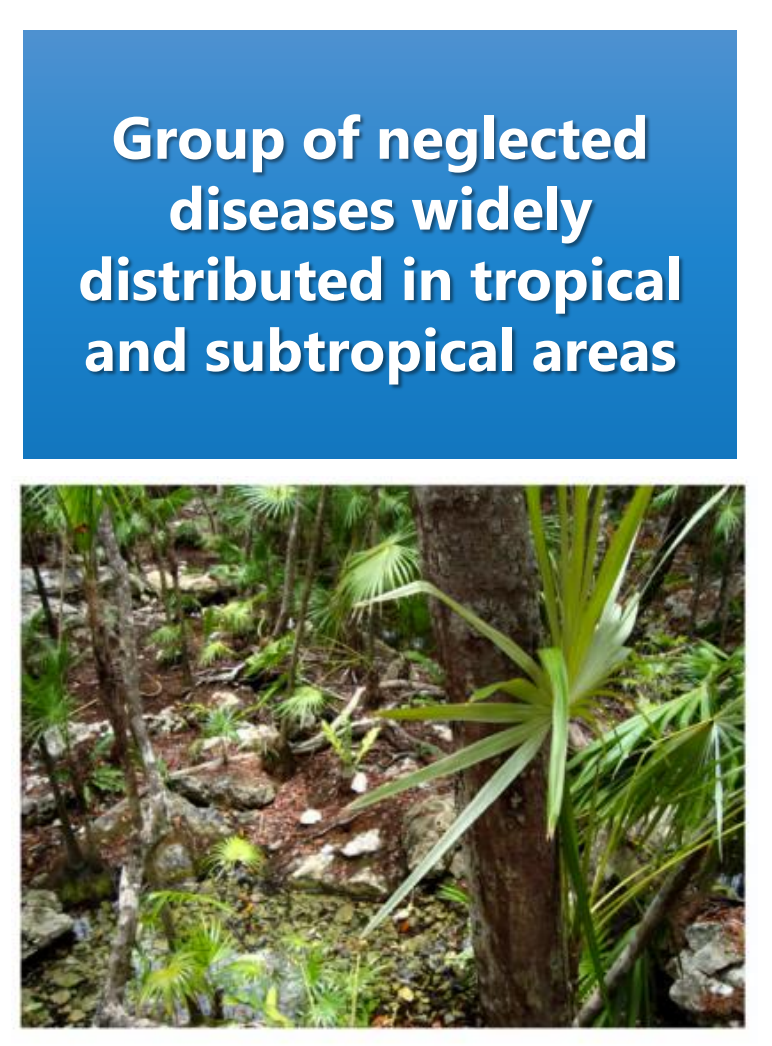

Caused by an obligate intracellular protozoan kinetoplastid from the genus Leishmania

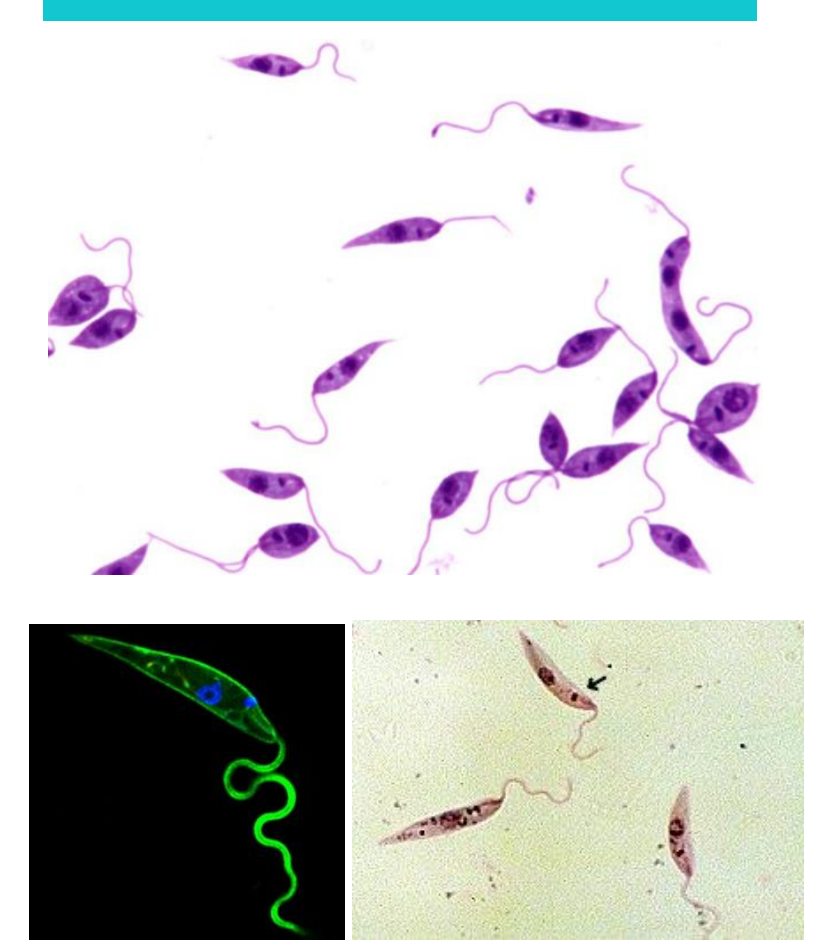

The infective form is transmitted to the human host by the bite of phlebotomineous females

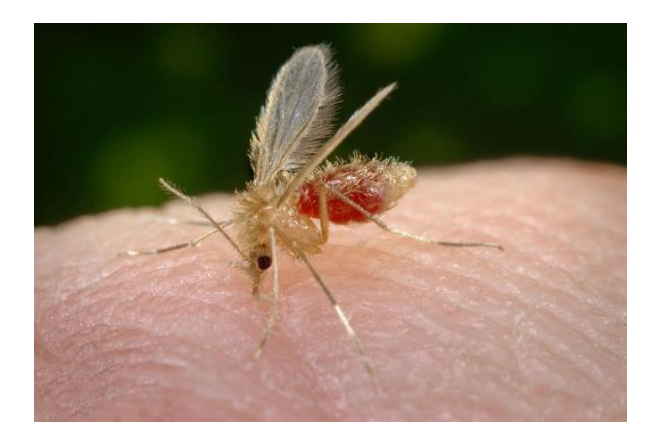

Vector: sandfly

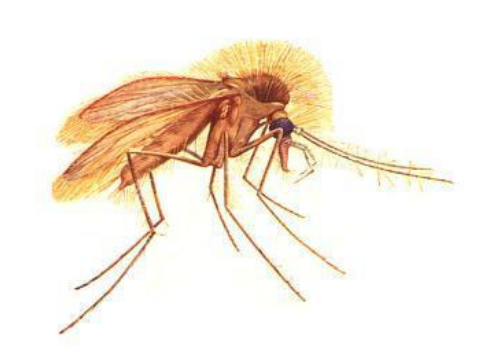

The clinical spectrum of the disease varies depending on the species involved and the immunogenic status of the host

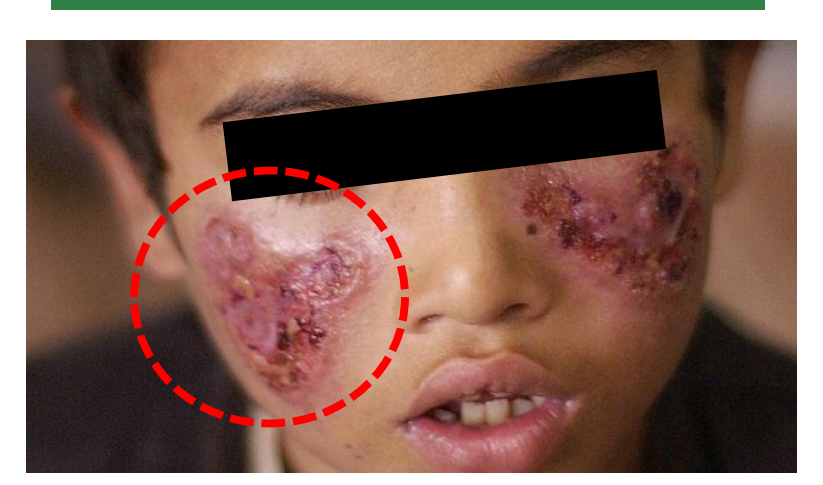

Child with cutaneous leishmaniasis

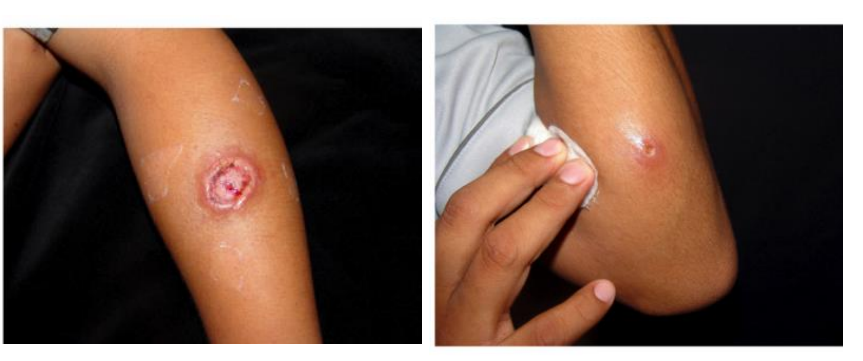




\section{Leishmaniasis: a public health problem in Colombia}

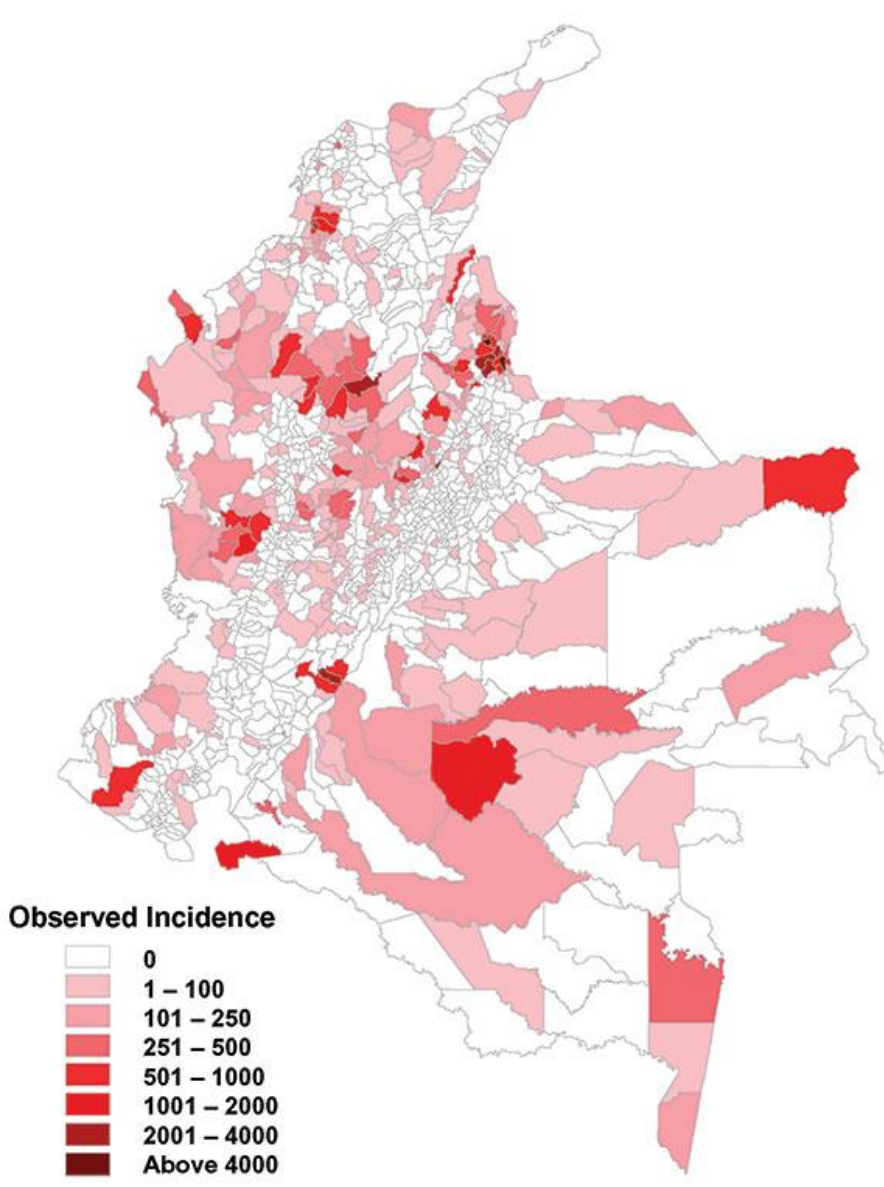

Geographic distribution of the incidence of cutaneous leishmaniasis by municipality (1994)

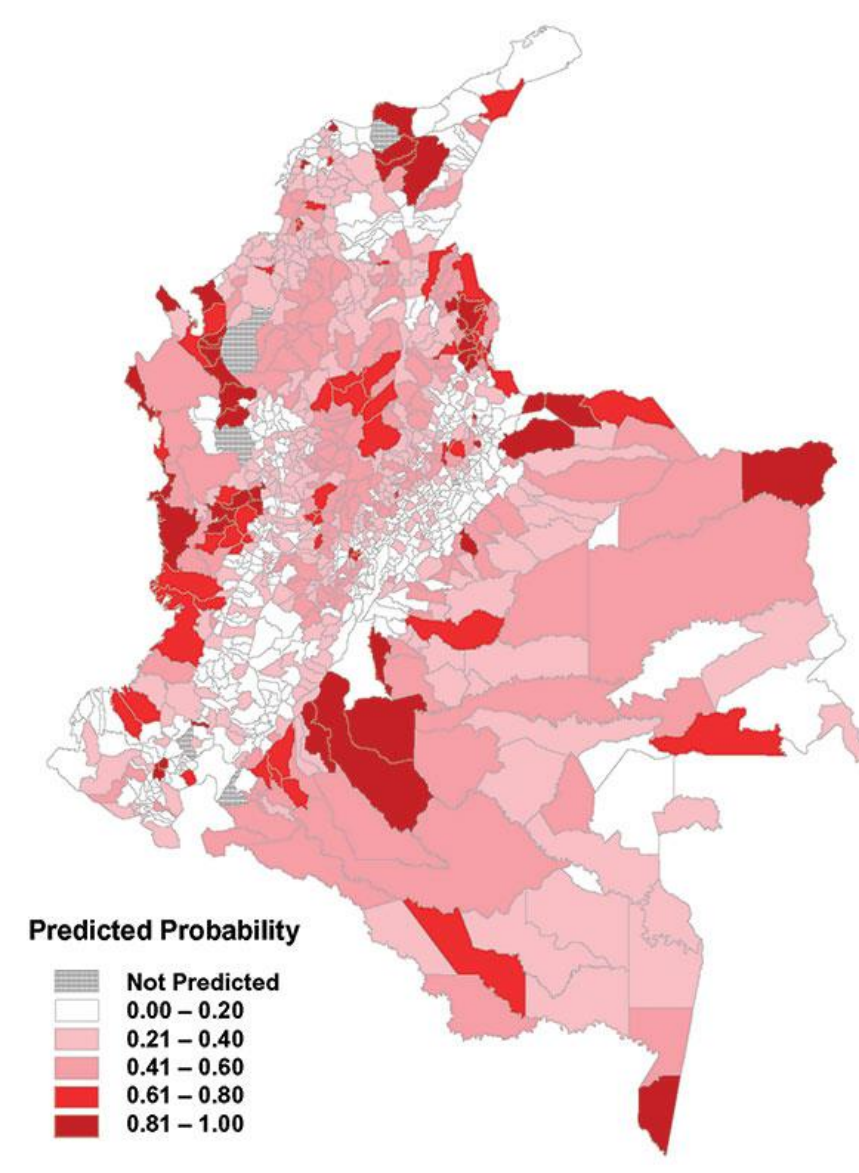

Map of predicted risk for the probability of transmission (2004)
Colombia is an endemic country for leishmaniasis

\section{More than a half} Colombians are at risk of contracting the disease
Colombia is one of the ten countries with the highest number of cases of cutaneous leishmaniasis

Approx. 65K new cases of cutaneous

leishmaniasis are estimated annually in Colombia
CUTANEOUS Leishmaniasis

Imillion

cases reparted in the last 5 years 


\section{Leishmaniasis: a public health problem in Colombia}

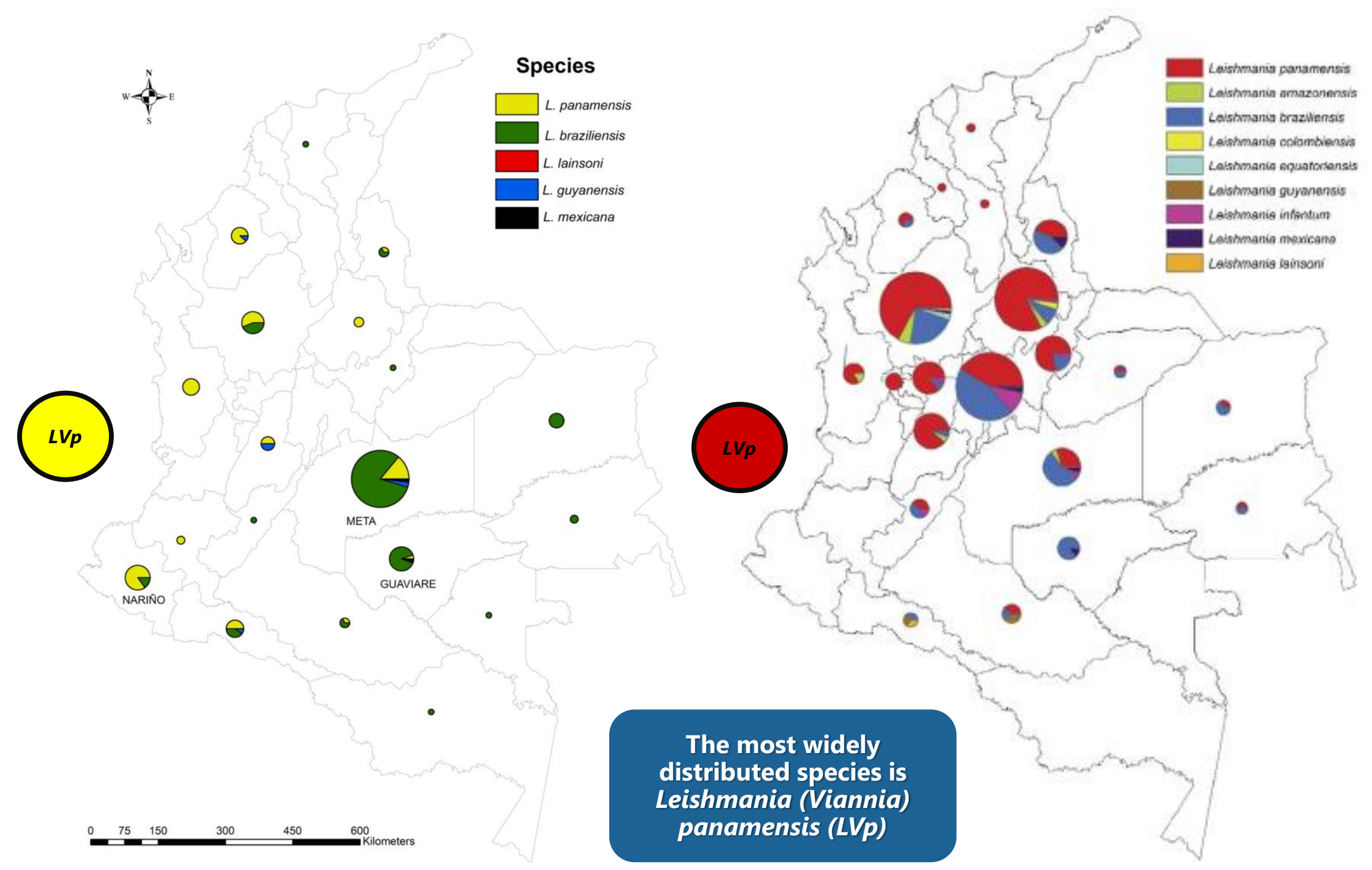

Treatment: chemotherapy with antimonial compounds, pentamidine or amphotericin B

Complicated, expensive, inefficient (it affects hard-toreach poor population)

There is only one oral drug (miltefosine) but increasing resistance has been reported

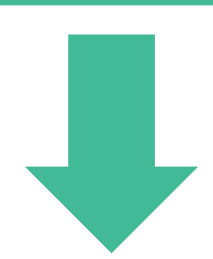

\section{A safe and effective} vaccine is needed 


\section{Leishmaniasis: vaccine development}

What are the immune mechanisms that lead to a protective response?

Infection model with L. major Th1/Th2 paradigm
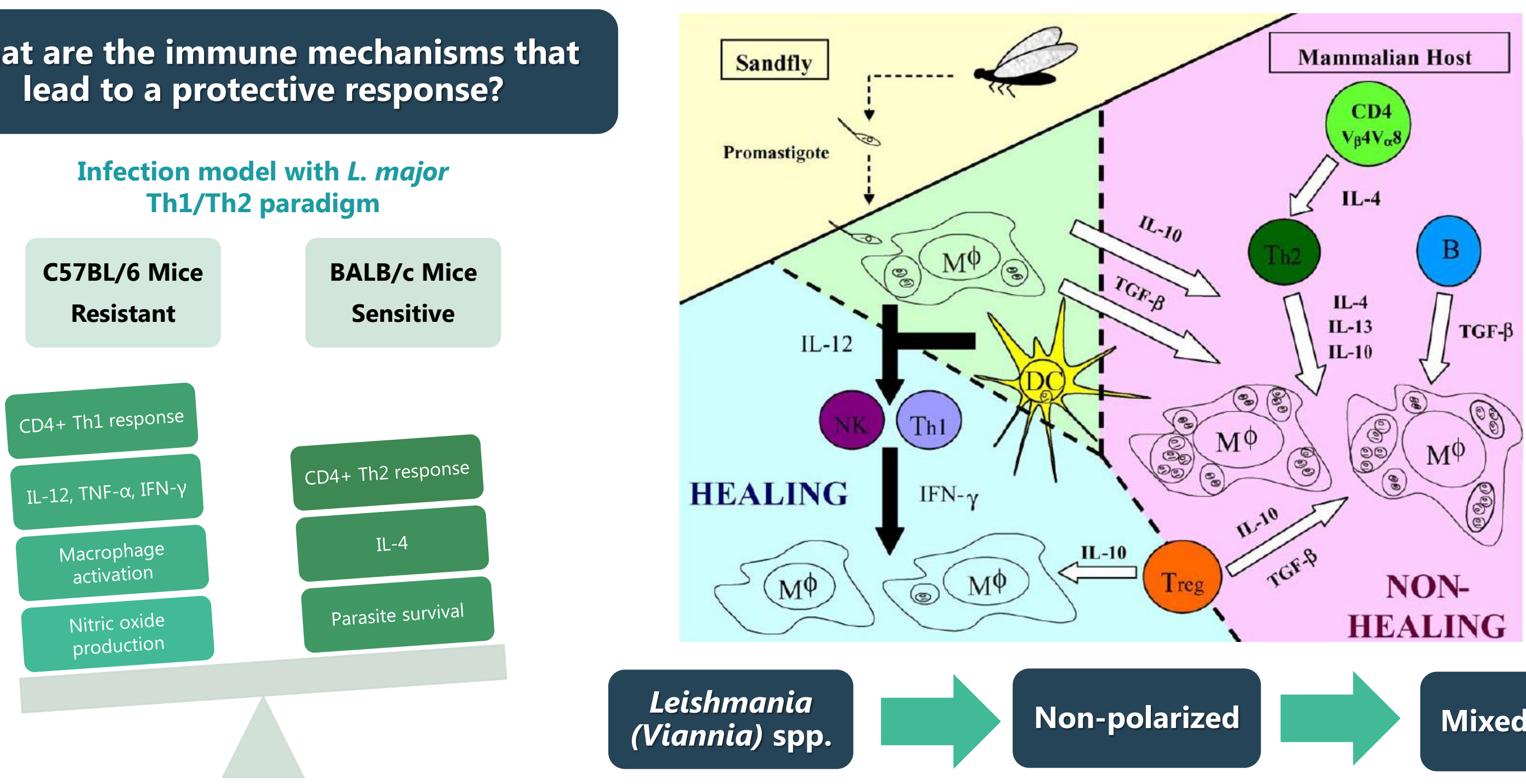


\section{Leishmaniasis: vaccine development}

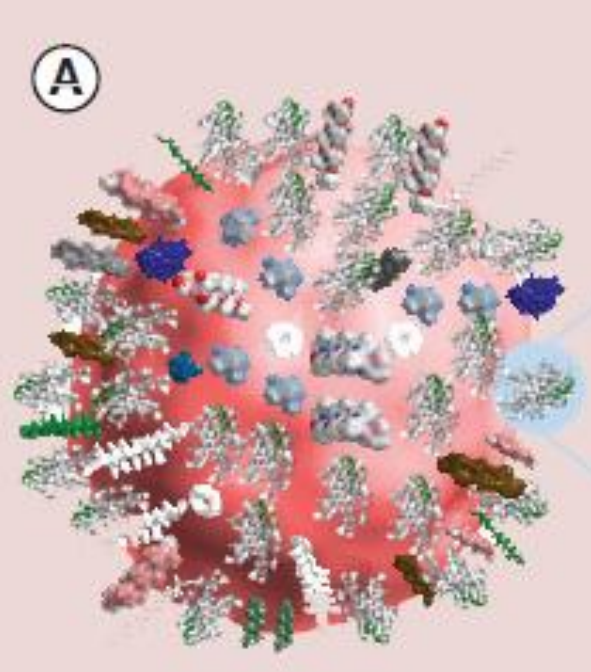

Whole pathogen
(B)

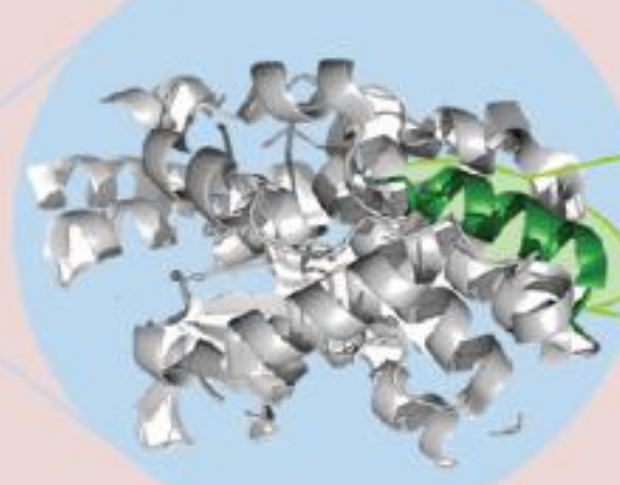

Protein
(C)

(D)

\section{Immunogenicity}

Side-effects 


\section{Components of a vaccine}

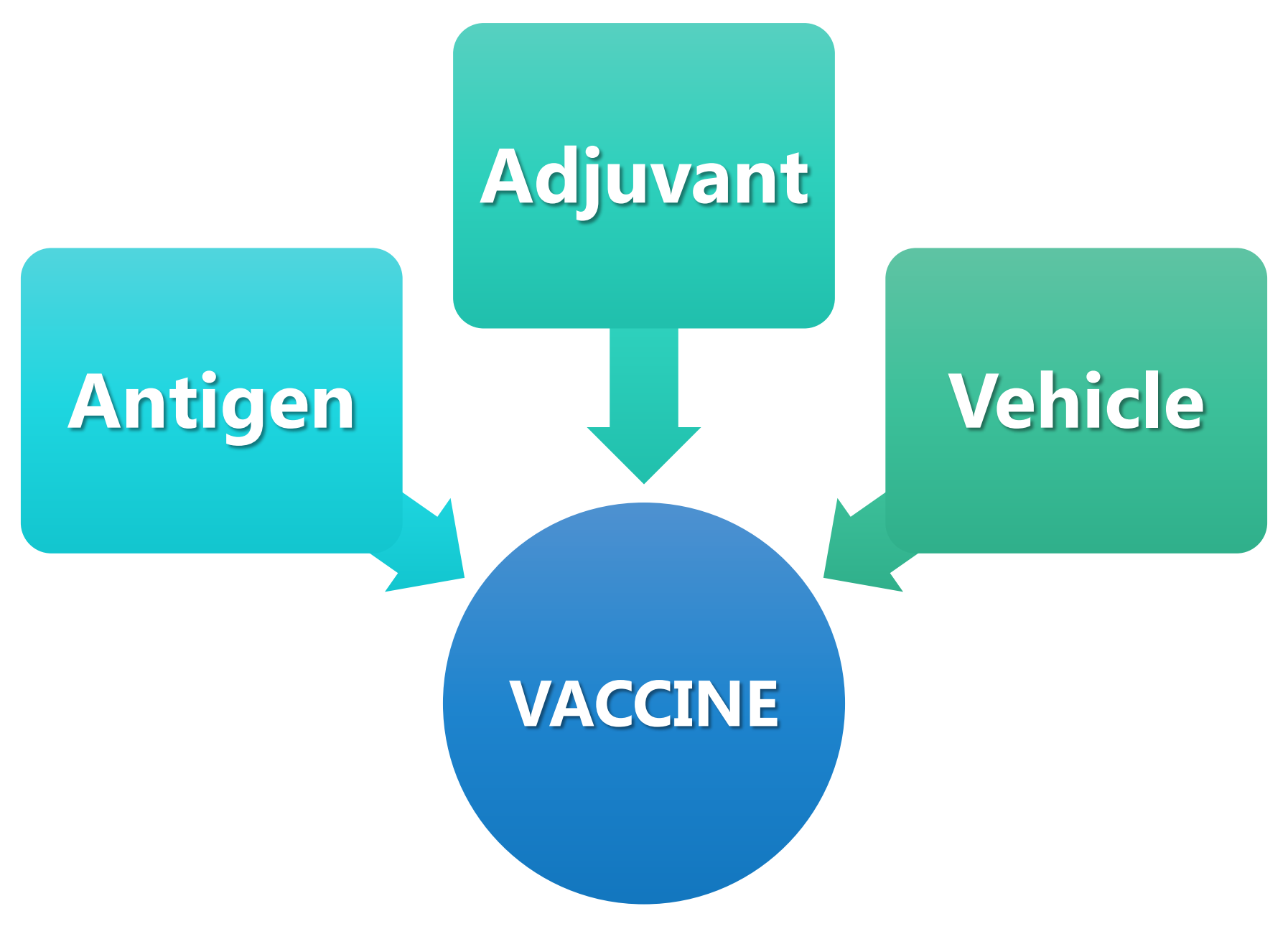




\section{Trypanothione Reductase as vaccine candidate}

TR from $L$. infantum

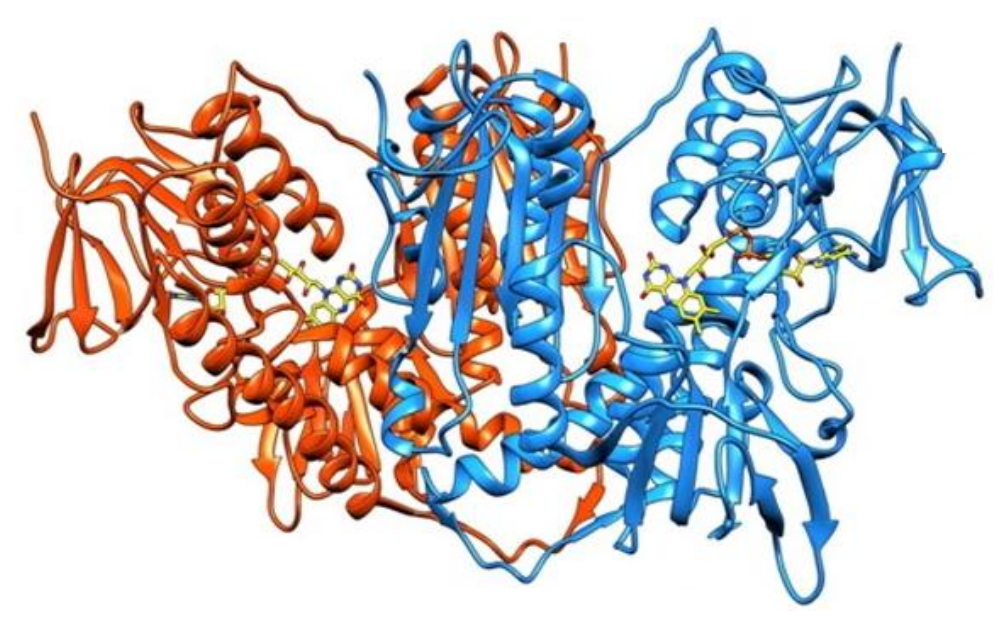

Occurs in protozoan parasites of the genus Trypanosoma and Leishmania
Oxidjoreductase enzayme

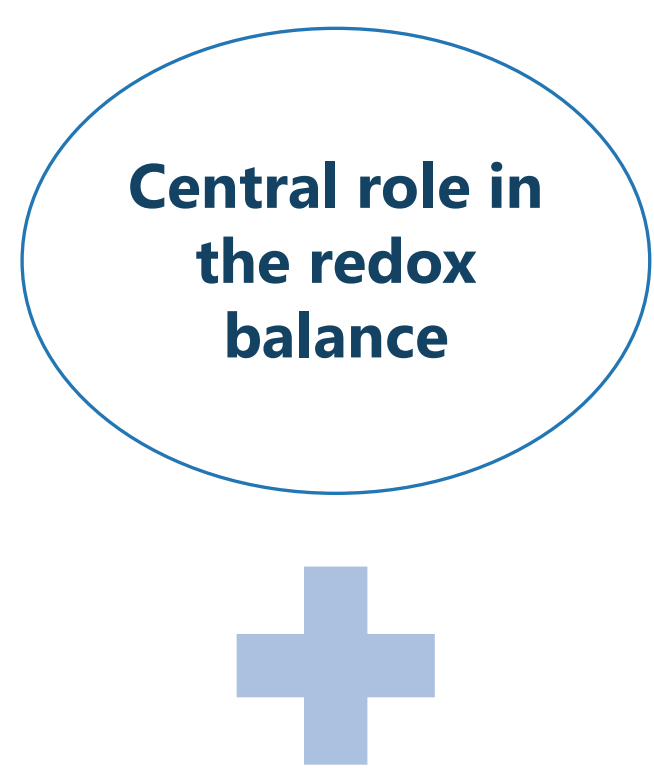

\section{Essential for} the infectivity of the parasite

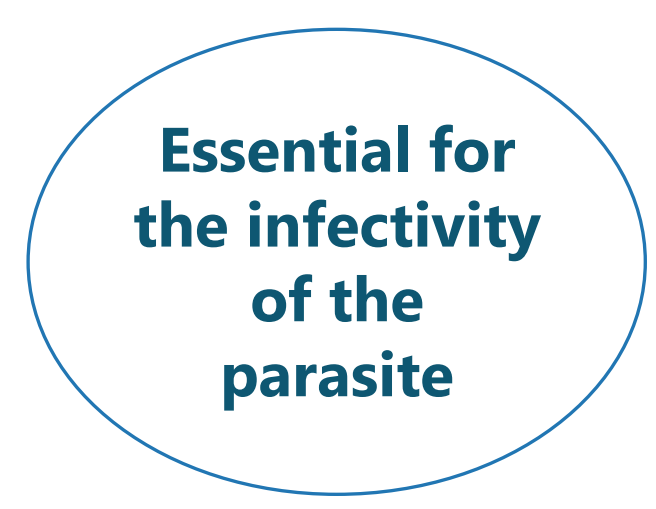

Pharmacological target

(development of inhibitory drugs)

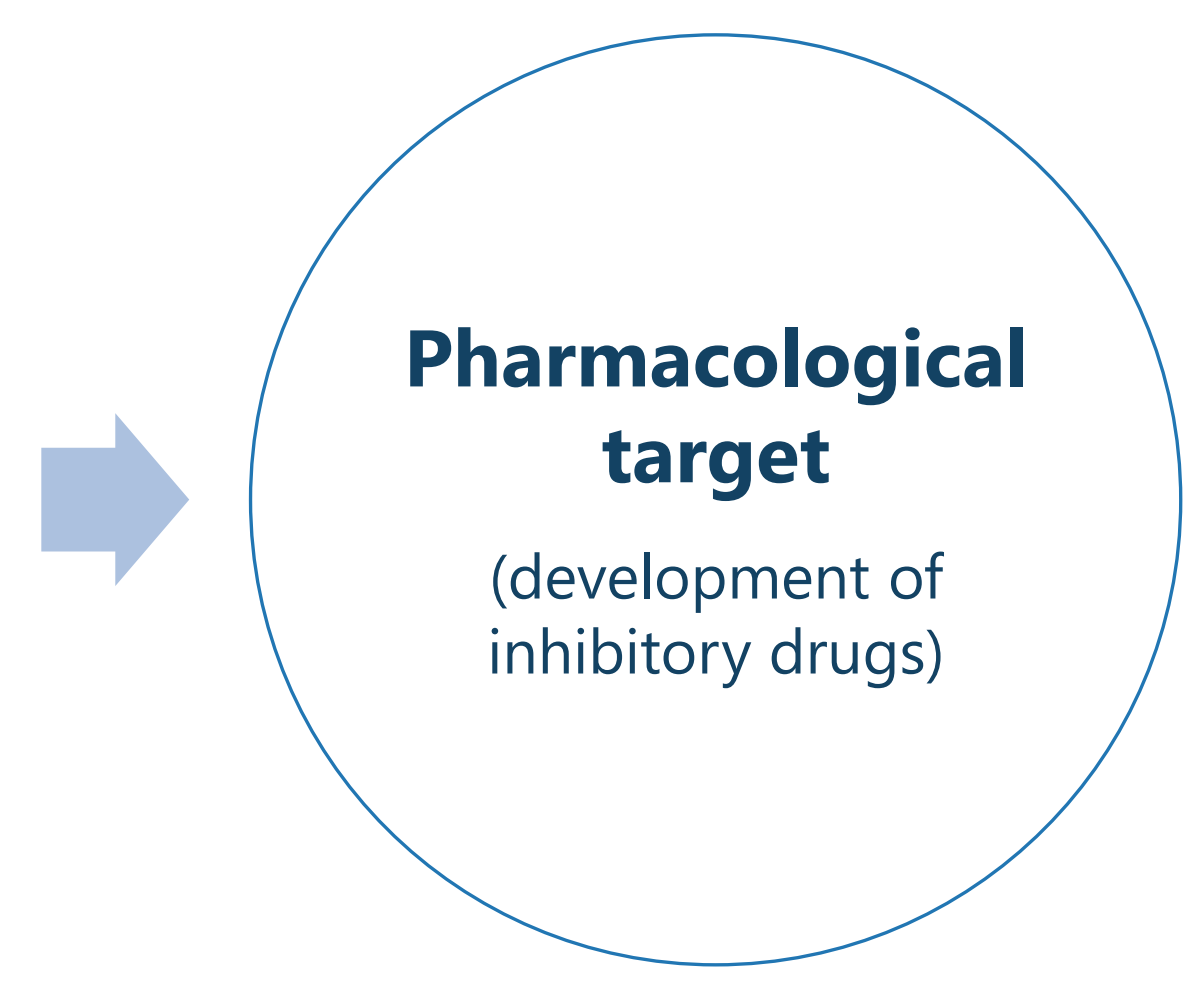

What about its immunogenic role? 


\section{Trypanothione Reductase as vaccine candidate}

\section{Evaluation of rTR immunogenicity}

$\checkmark$ Peripheral blood mononuclear cells (patients)

$\checkmark$ Lymph node cells (infected and treated hamsters)

After stimulation with $\mathbf{r T R}$, the proliferative response was comparable or superior to the stimulation with soluble antigen

\section{Evaluation of the prophylactic efficacy from rTR \\ Hamsters \\ $\checkmark$ BCG as adjuvant \\ $\checkmark$ VL model (infection by Leishmania donovani)}

\section{Clinical (up to 6 months) \\ (1060\% protection against infectious challenge. \\ (10) Weight gain. \\ (10Healthy condition.}

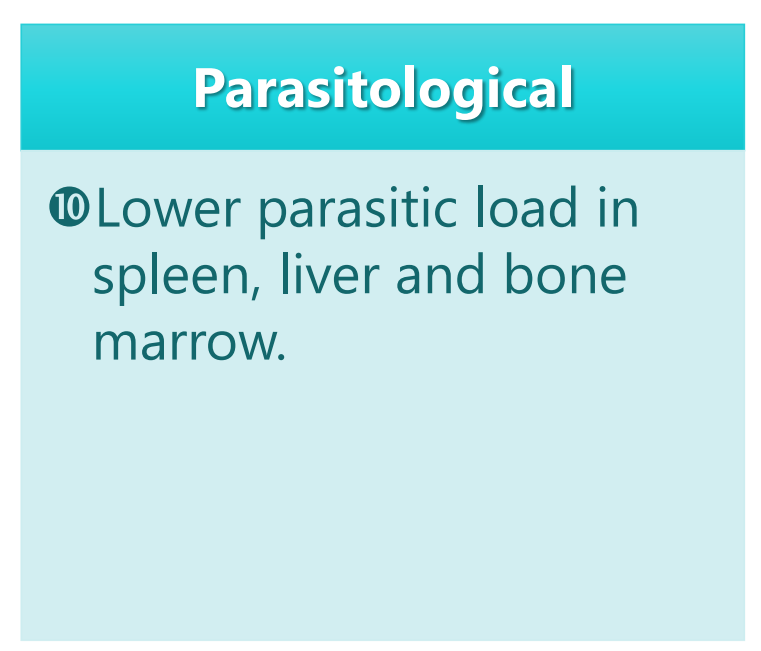

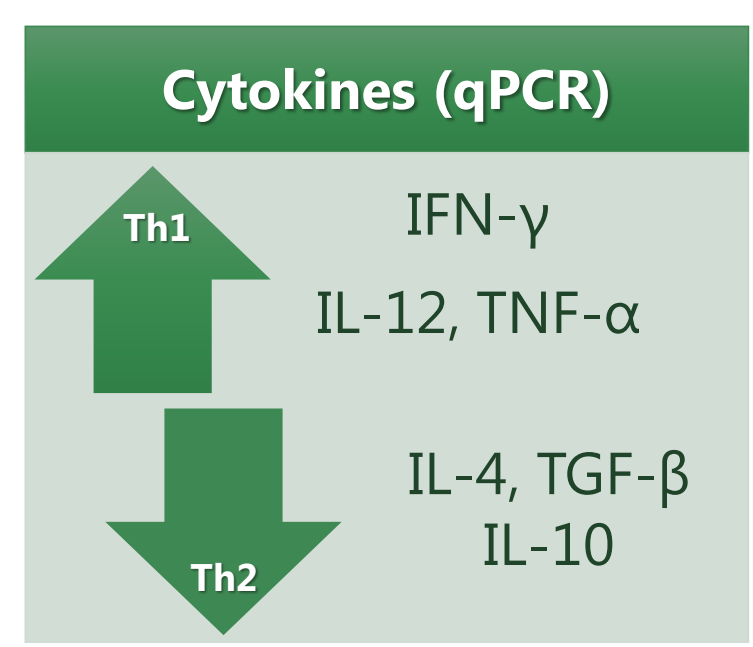

The design of polyvalent chimeric vaccines is suggested but an encapsulation system is not proposed 


\section{Cellular adjuvants for vaccines}
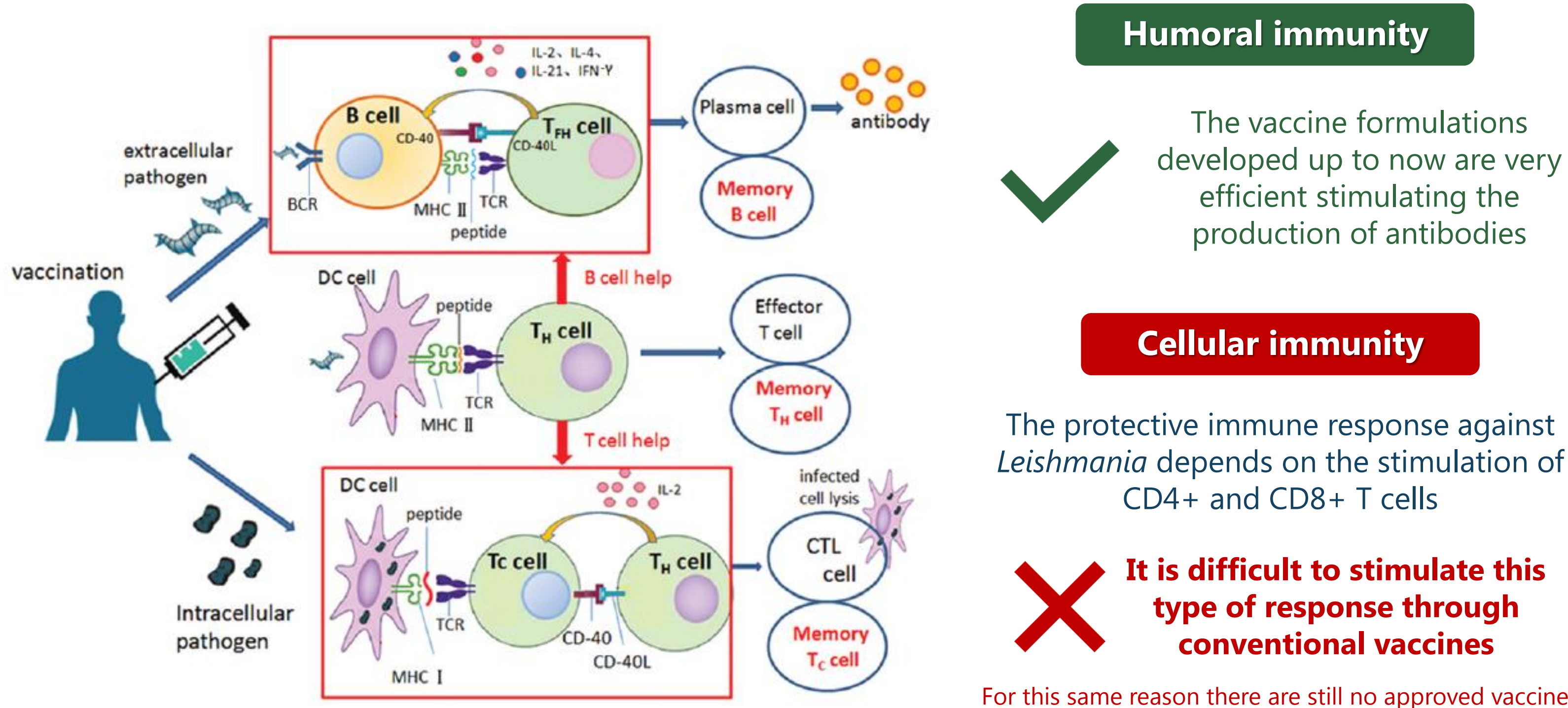

For this same reason there are still no approved vaccines against HIV, malaria, tuberculosis and cancer 


\section{Cellular adjuvants for vaccines}

\section{Receptors of $\checkmark$ TLR innate $\quad \checkmark$ NLR immunity $\checkmark$ LTR
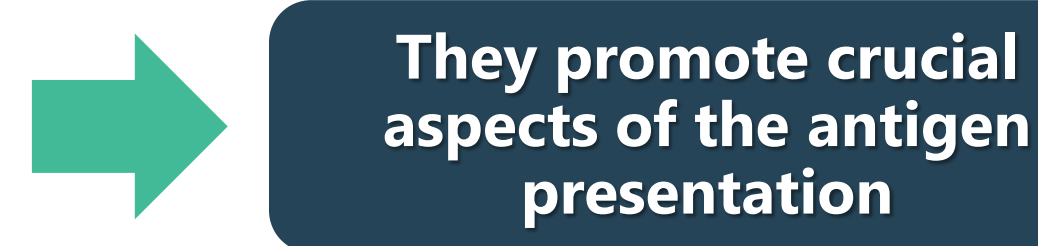
aspects of the antigen presentation \\ $\checkmark$ Antigen capture and processing \\ $\checkmark$ Dendritic cell maturation}

\section{Non-methylated} CpG motifs

TLR9 ligand

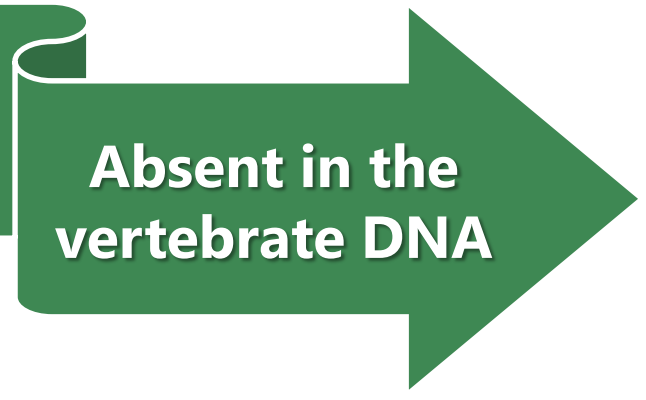

CpG ODN Classes

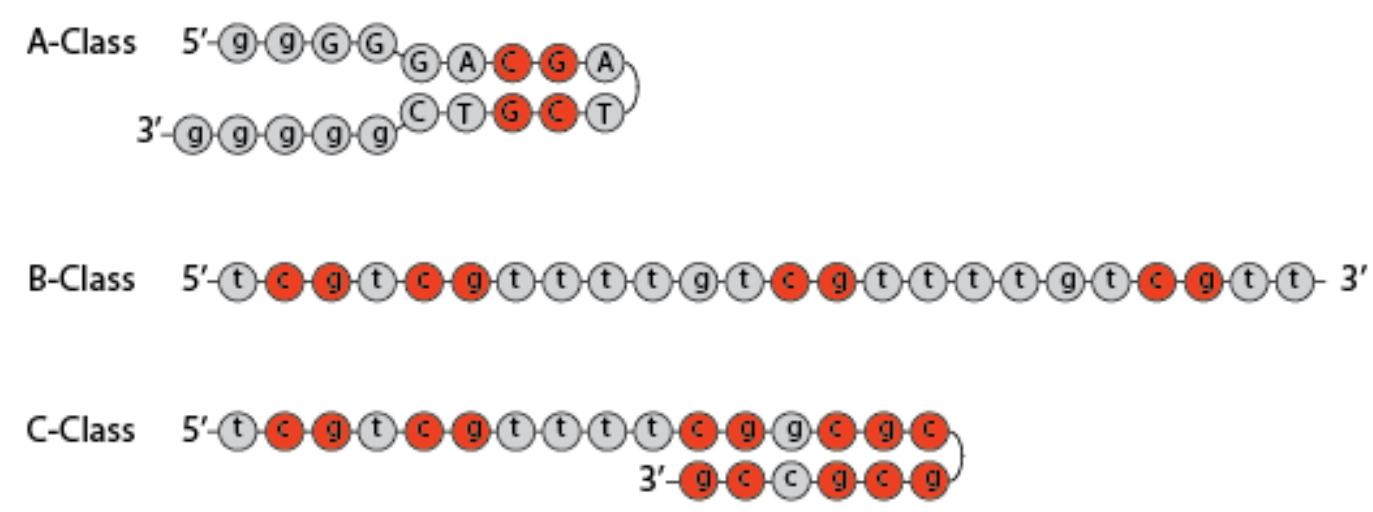

(I) Phosphorothioate link, (N) Phosphodiester link, C-9 CG dinucleotide https://www.invivogen.com/cpg-odns-classes 


\section{Liposomes as adjuvants/delivery system for vaccines}

Micro/nanometric spherical vesicles with at least one lipid bilayer composed of phospholipids

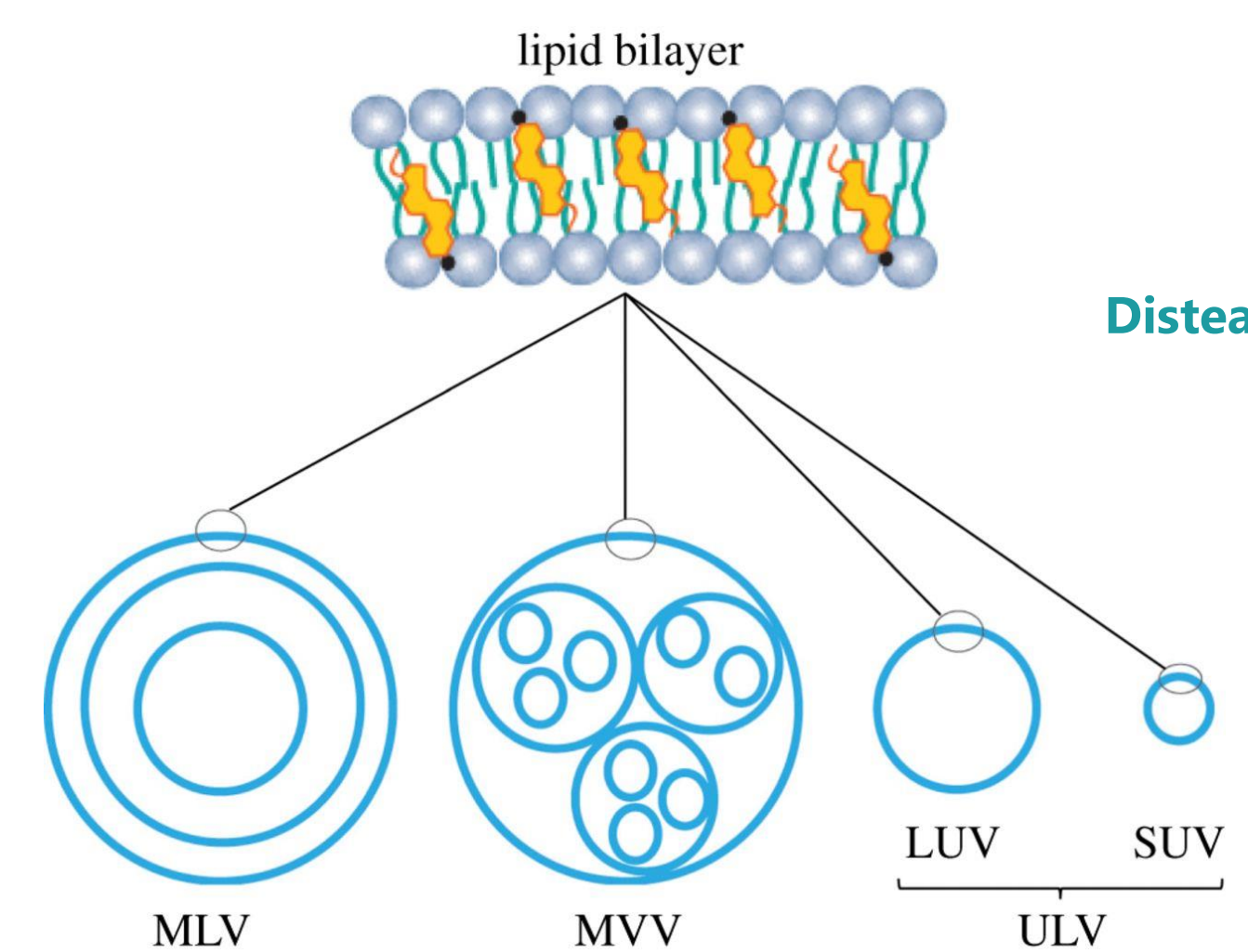

Tunable physicochemical characteristics

\section{Main lipid}

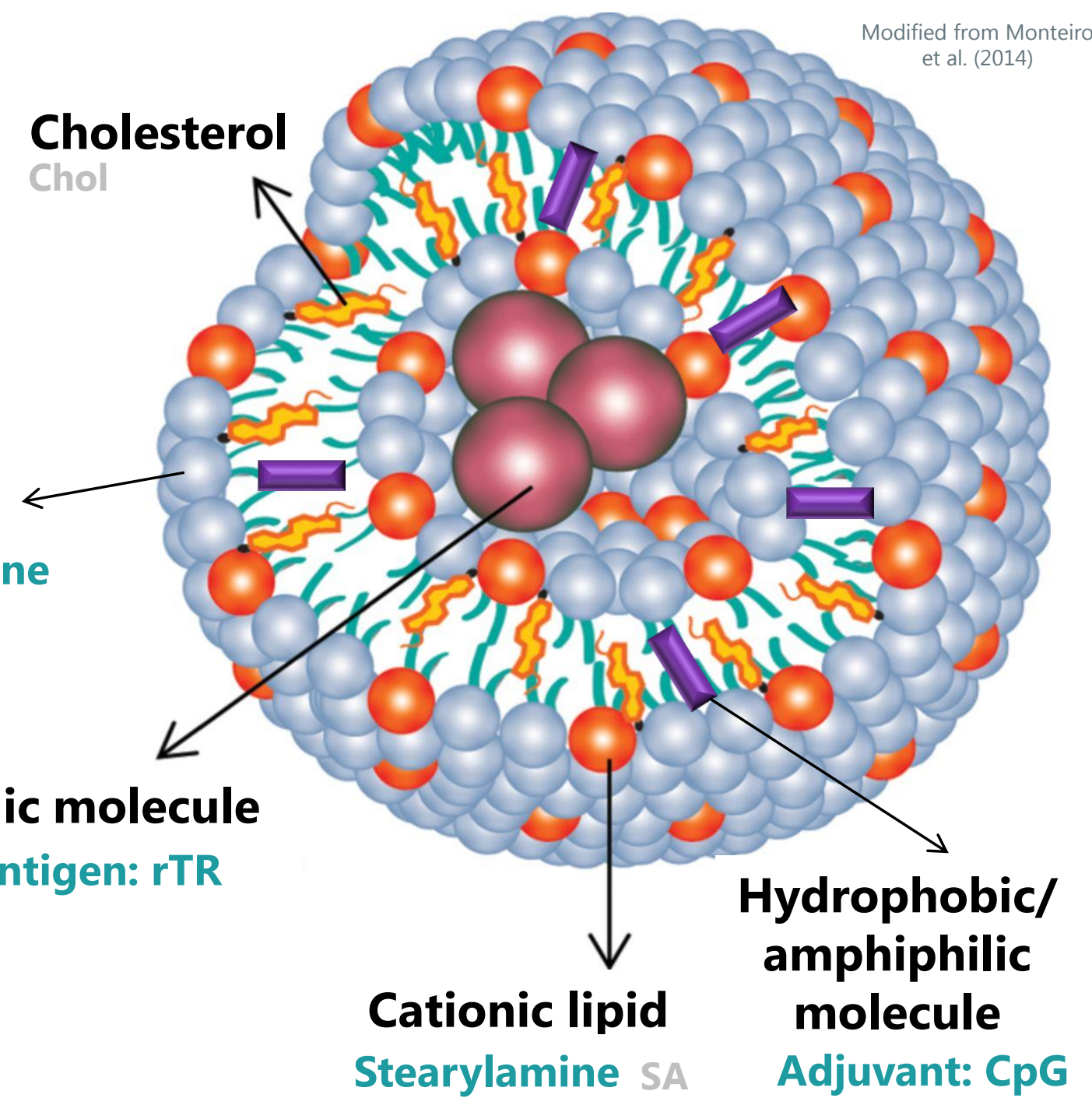




\section{Liposomes as adjuvants/delivery system for vaccines}

\section{Depot effect}

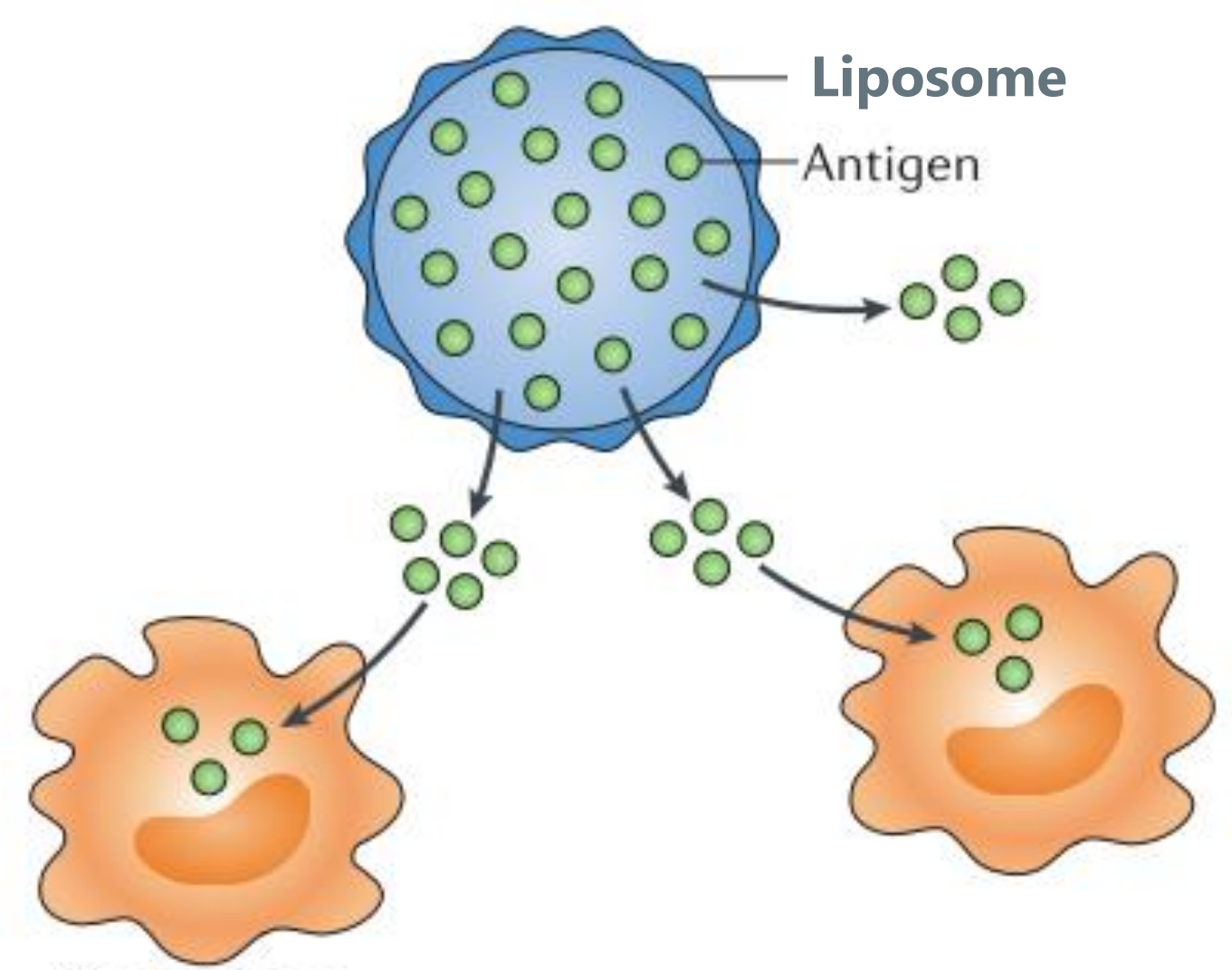

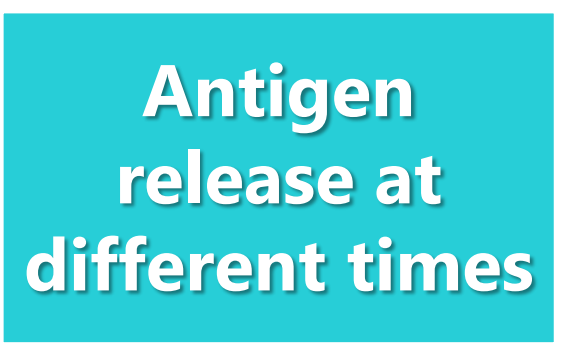

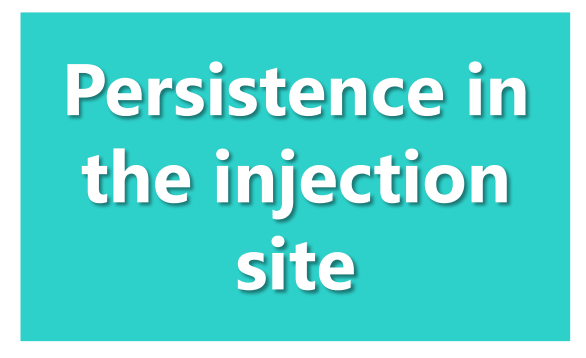

It depends on the particle size

Macrophage
Activation by intense presentation




\section{Liposomes as adjuvants/delivery system for vaccines}

Example antigen density

Effective

(extracellular)

concentration

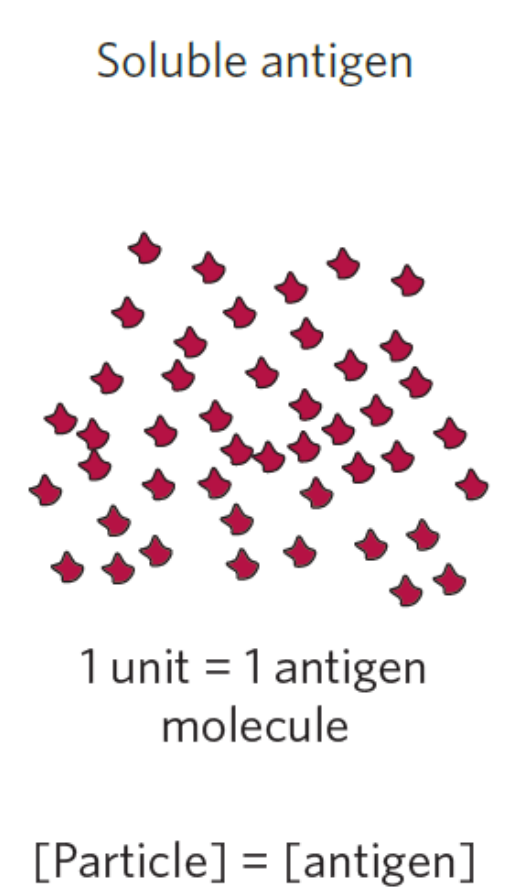

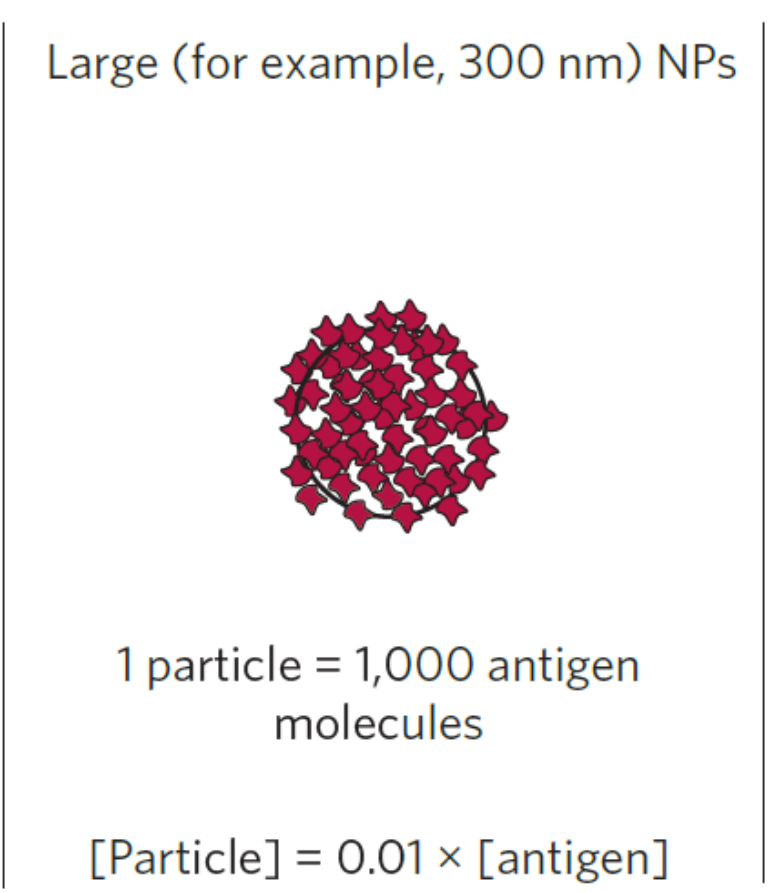




\section{Liposomes as adjuvants/delivery system for vaccines}

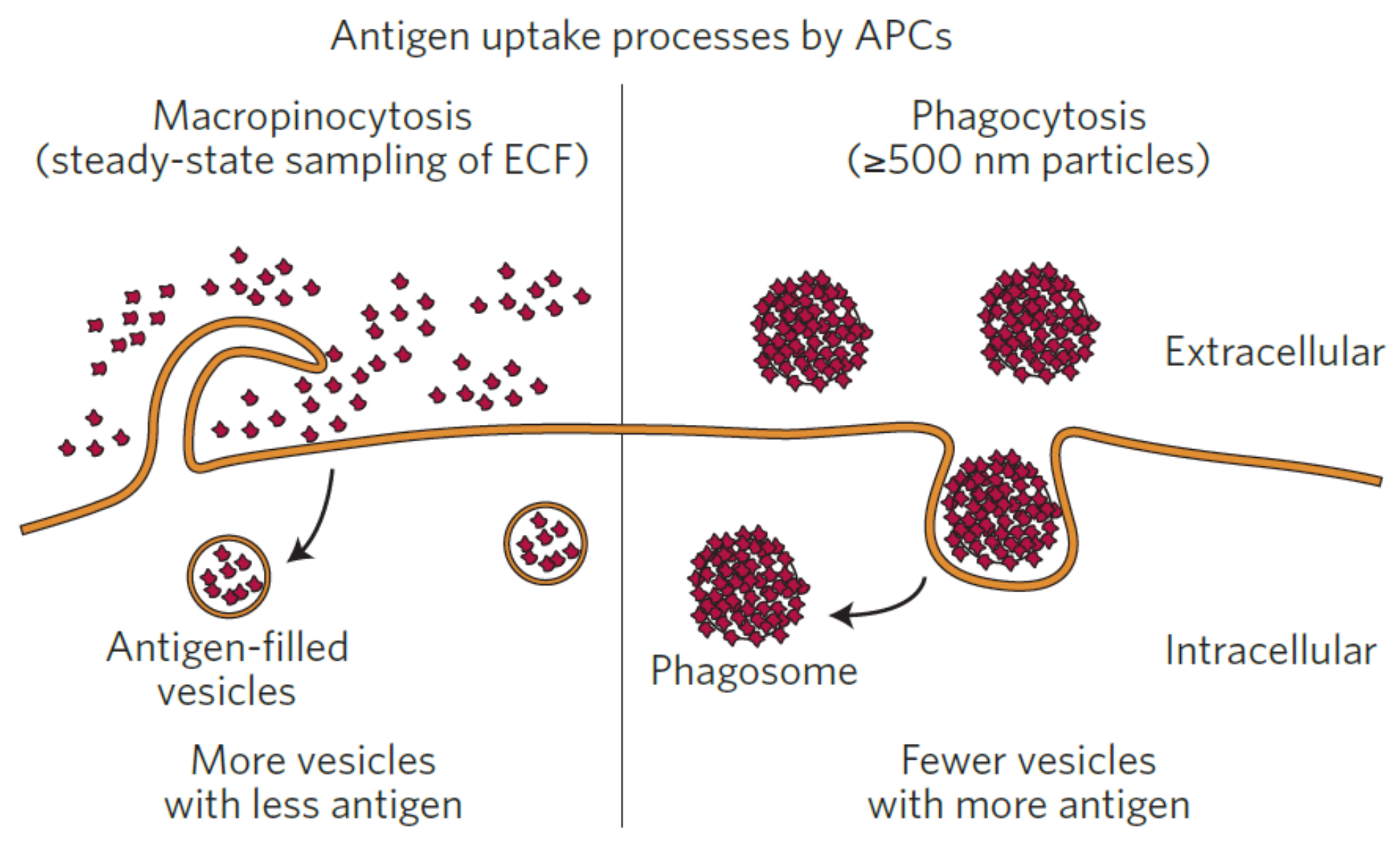




\section{Liposomes as adjuvants/delivery system for vaccines}

Taking and trafficking of liposomal antigens

a Delivery of antigens
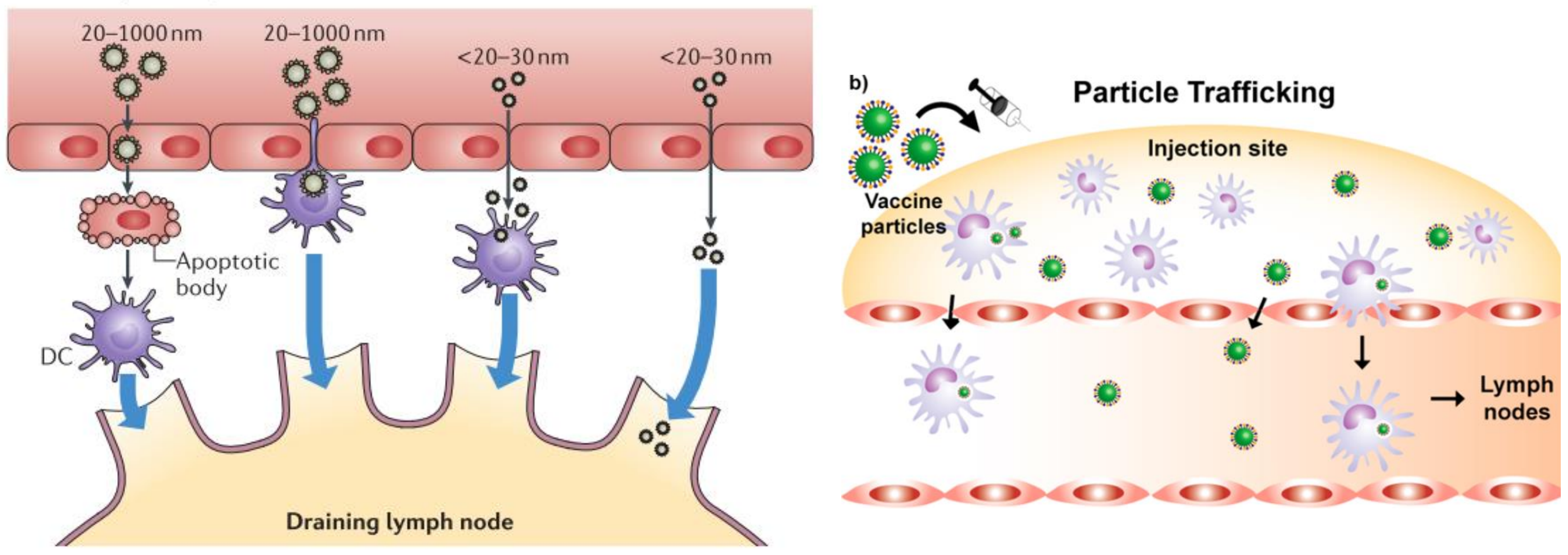


\section{Liposomes as adjuvants/delivery system for vaccines}

c Repetitive antigen display

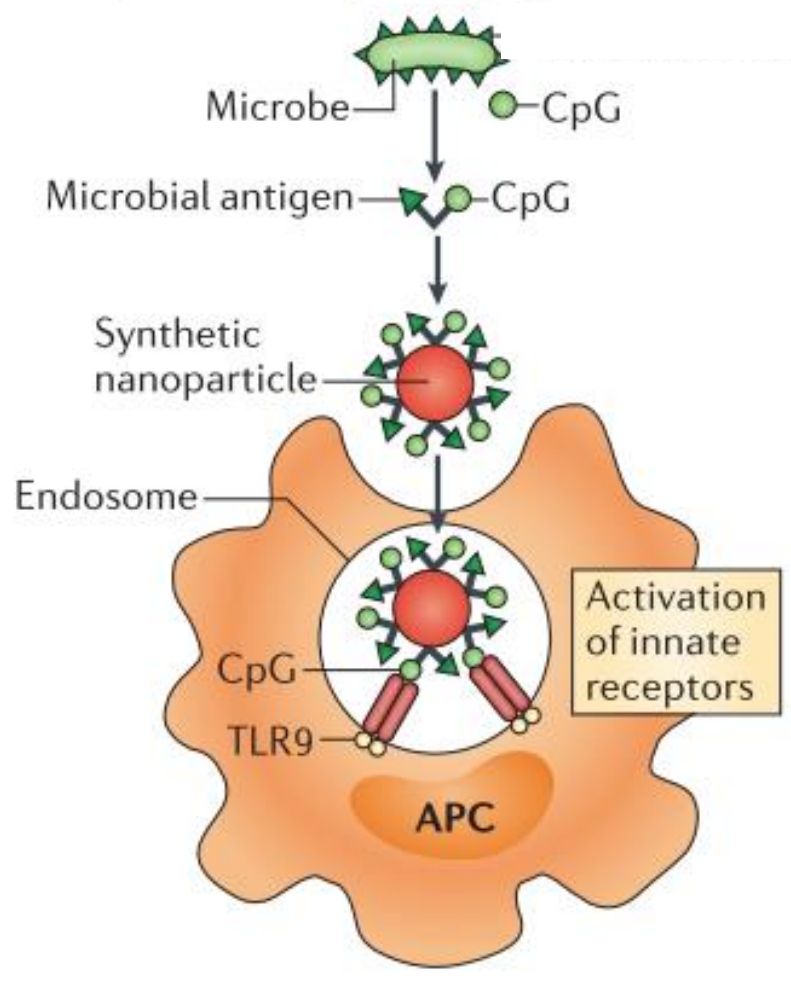

Processing of

phagocytosed antigen and presentation to $\mathrm{T}$ cells

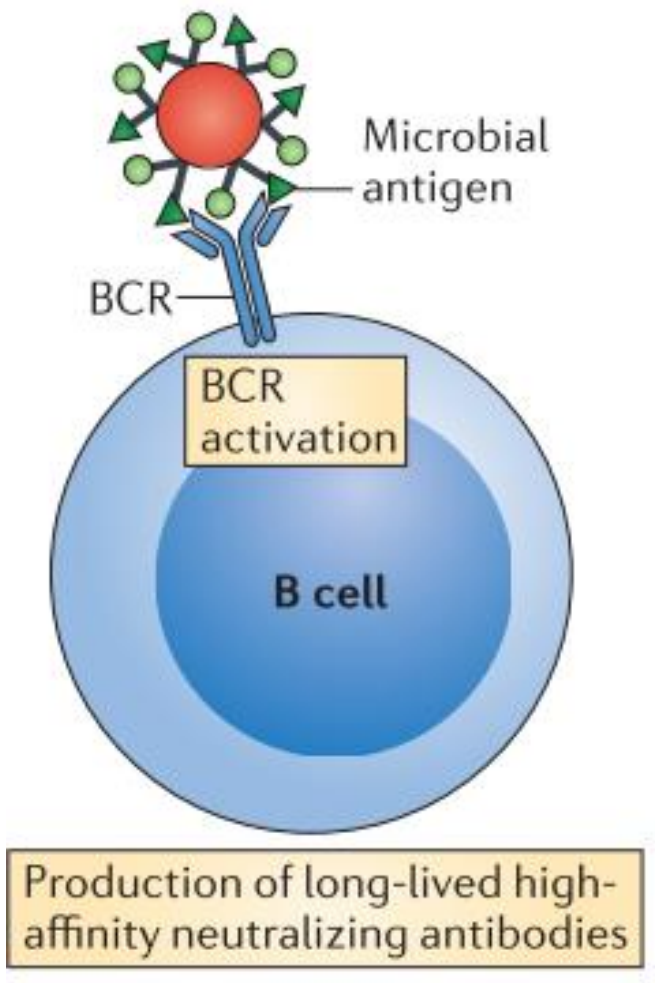

d Cross-presentation

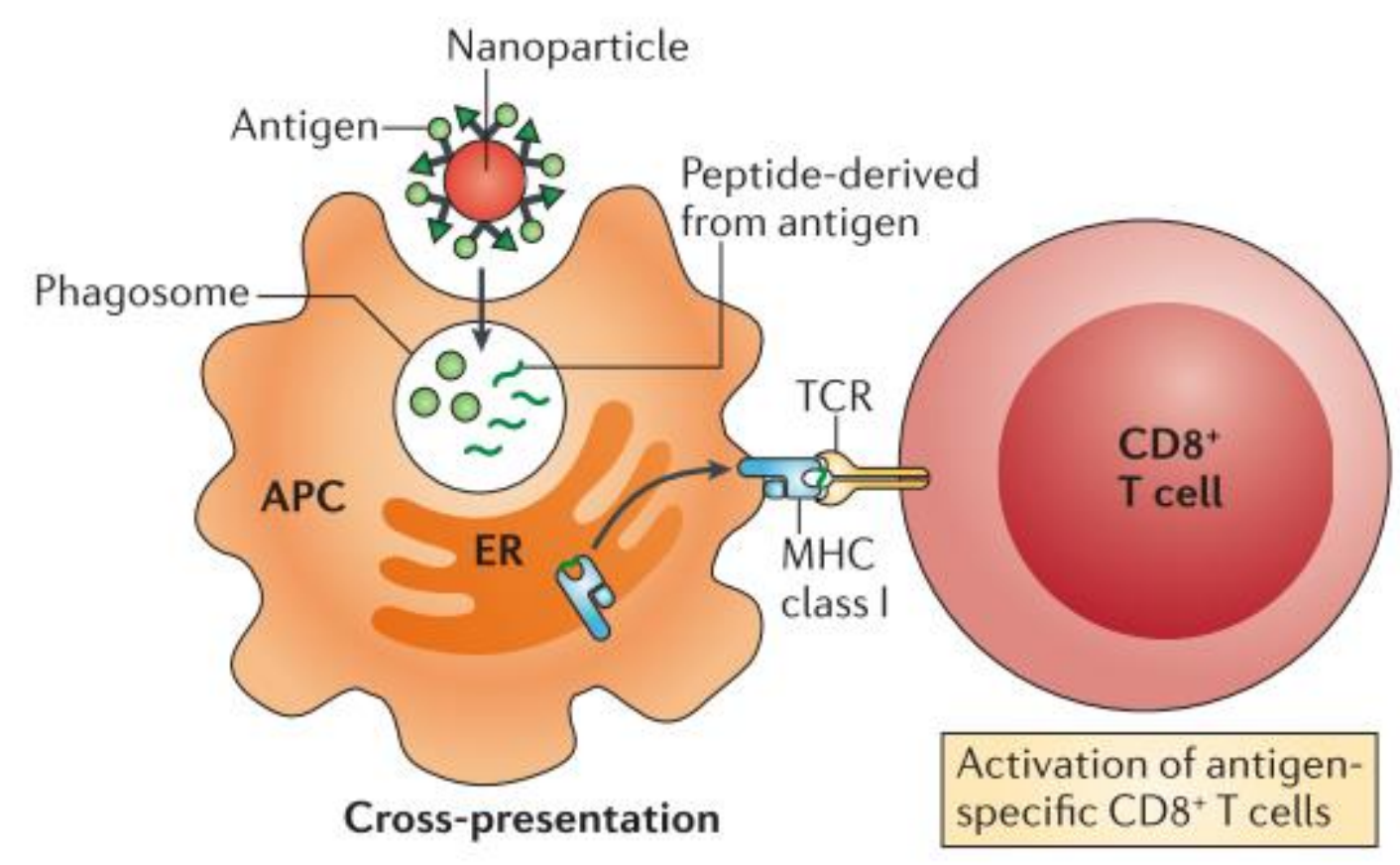

Presentation of the

intact antigen to $B$ cells
Cross-presentation to $丁$ lymphocytes 


\section{Subunit liposomal nanovaccines to prevent leishmaniasis}

Infections caused by $L$. major and $L$. donovani
Mainly liposomes of total protein antigen
Several purified proteins have been found to be protective
Some have been encapsulated in liposomes
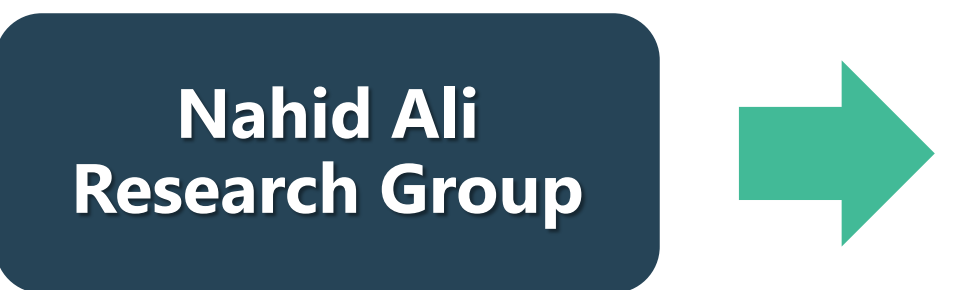

Subunit vaccine to prevent VL in India
Antigens encapsulated in rigid cationic liposomes are more efficient
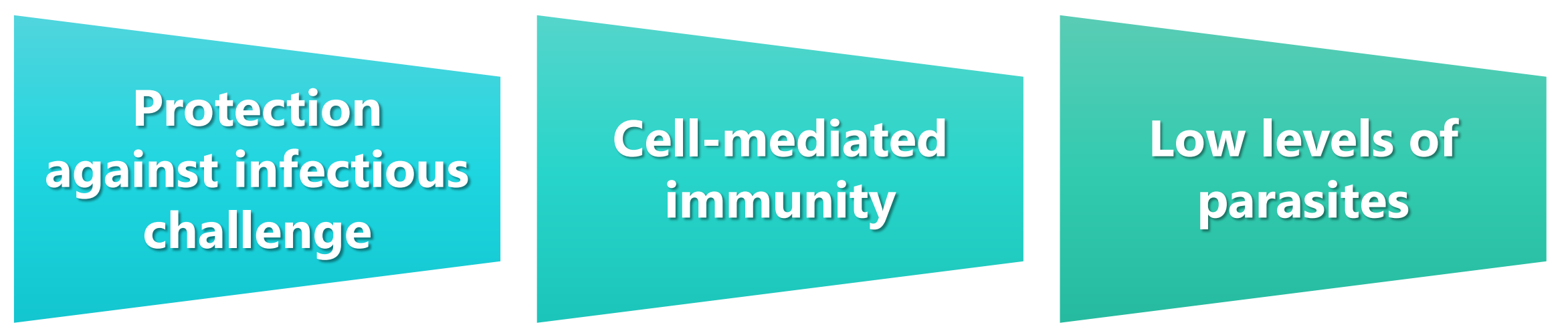

Stimulation of Th1 response 


\section{BACKGROUND}

Our ulcerative murine model of infection with $L V p$ reproduces the human leishmaniasis characteristics

Synthetic ODN with CpG motifs is a protective adjuvant when combined with the total lysate of the parasite

Our liposomal formulation of soluble antigen from Leishmania induces protection against infectious challenge

TR is a promising antigen to formulate a molecularlydefined vaccine 


\section{HYPOTHESIS}

Vaccination with a micro/nanostructured formulation (cationic liposomes) of rTR, either individually or in combination with CpG, potentiates the specific immune response needed to protect mice from an infectious challenge in the model of cutaneous leishmaniasis caused by Leishmania (Viannia) panamensis

\section{GENERAL OBJECTIVE}

To evaluate the prophylactic efficacy of both soluble and micro/nanostructured formulations of the rTR with/without CpG (soluble/liposomal) 


\section{METHODOLOGY Production of rTR}

National Center for Genomic Sequencing

\section{Optimization of factors} such as temperature, time and inductor concentration $\checkmark$ Expression vector: pET28a (+)

$\checkmark$ Heterologous expression system: Escherichia coli (DE)

Solubility evaluations by lysis method by sonication and separation by centrifugation

Desalting and gel filturation chromatography
Protein labeled with polyhistidine-tag (His6-Tag)
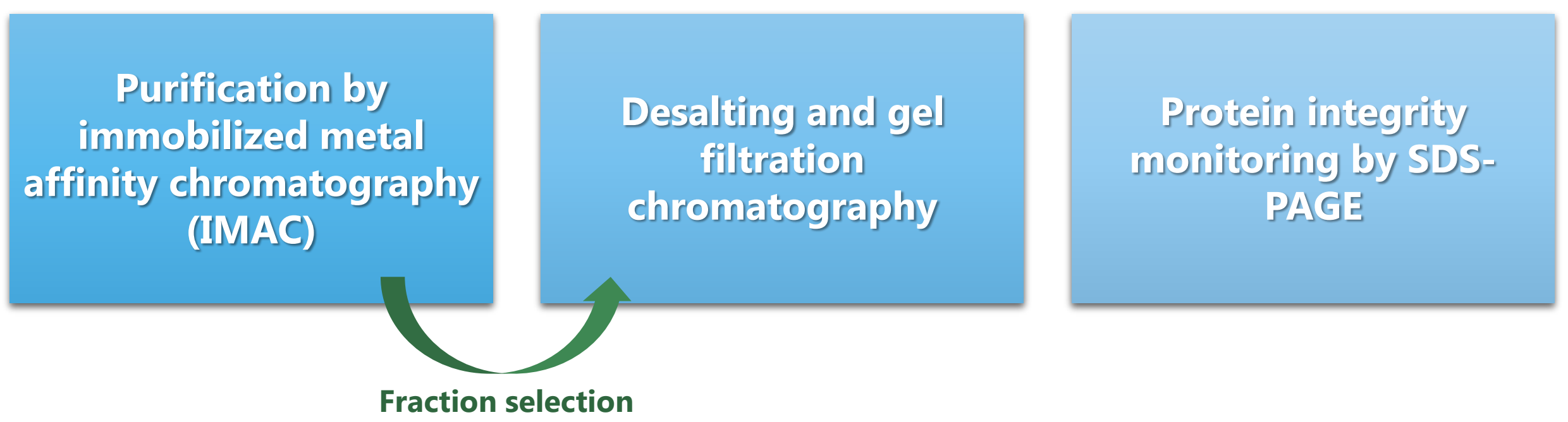


\section{METHODOLOGY Preparation and characterization of liposomes}

\section{Conventional thin lipid fildm hydration technigue}

Dissolution of hydrophobic compounds
Hydration of the lipid film

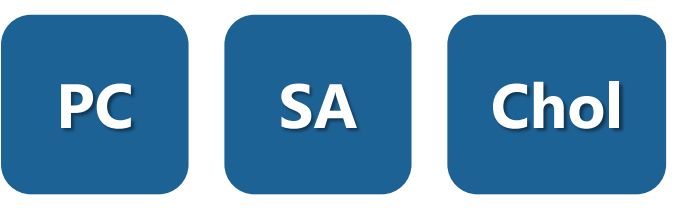

Size homogenization by extrusion

\section{0}

Creation of the lipid film

\section{Dispersion and} agitation
Separation by centrifugation
Physjical characterization

Chemical characterization $\checkmark$ Particle size (hydrodynamic radius) by dynamic light scattering $\checkmark$ Particle charge (zeta potential) by electrophoretic mobility

$\checkmark$ Quantification of the protein by SDS-PAGE / densitometry $\checkmark$ Quantification of the adjuvant by UV-Vis spectrophotometry 


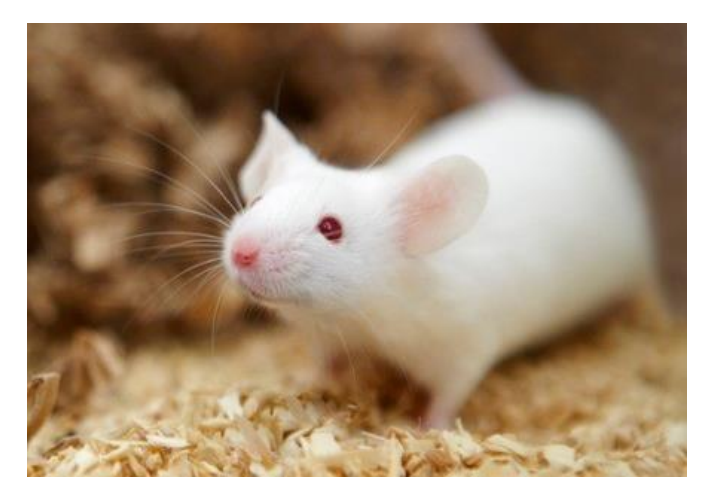

Female BALB/c mice 6-10 weeks old

SPF Animal Care Facility - SIU/UdeA
Lesion area

Score (0-4), the higher the number the more severe the injury Photographic record

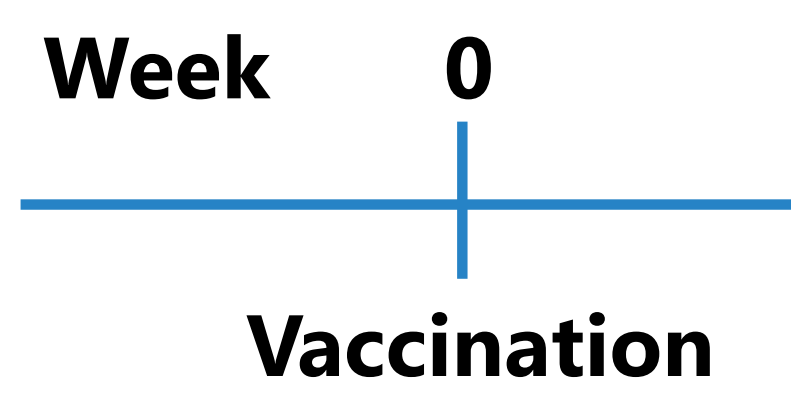

Subcutaneous injection

Tail base
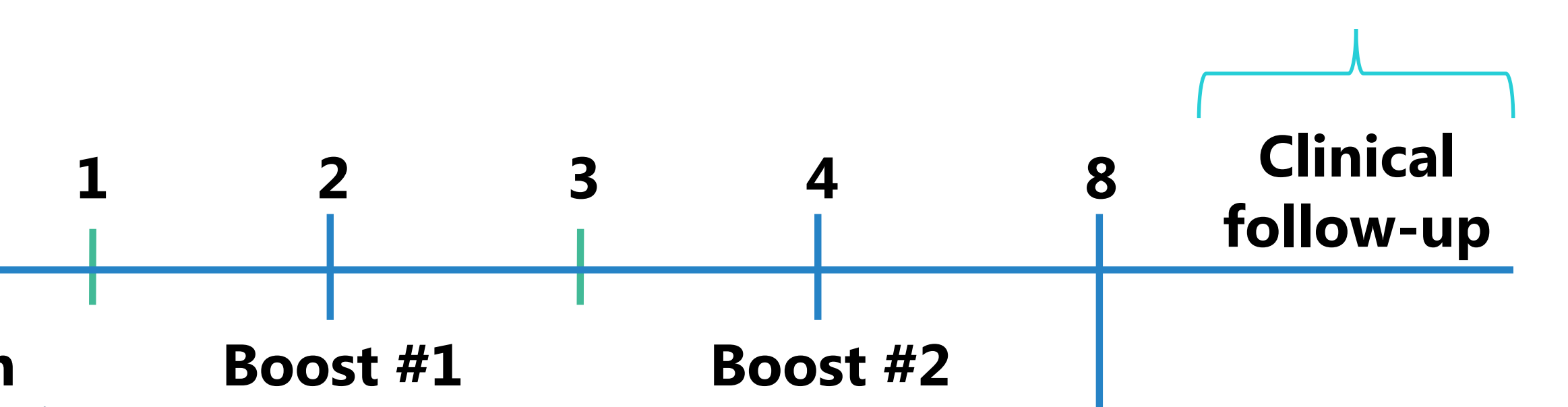

3

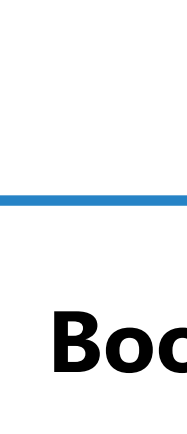

Clinical follow-up

\author{
Infection \\ $10^{5}$ LVp UA-946 parasites \\ Intradermal injection on ear
}




\section{METHODOLOGY}

\section{Parasitic load and antibody measurement}

\section{Parasjitic Joad Limiting dilution assay}

Mechanical disruption of the infected ears was performed in

supplemented Schneider medium
An initial dilution was performed and then 12 serial 1:3 dilutions were made in 96-well plates
Cultures were incubated for 4 weeks at $26^{\circ} \mathrm{C}$ and the growth of parasites was monitored weekly
Antibody measurement

Serum levels of IgG1 and IgG2a type antibodies were determined by ELISA

Overnight sensitization with rTR (antigen) was performed, then a blockade with BSA was made. Sera and, subsequently, antibody for each subclass of IgG was added
The addition of the chromogenic substrate tetramethylbenzidine (TMB) produced a colorimetric reaction whose absorbance was read at $655 \mathrm{~nm}$ 


\section{RESULTS Selection of quantification methods}

SDS-PAGE

UV-Vis Spectirophotometisy

Simple and reproducible

$\checkmark$ Calibration curve with standard protein or rTR in the same gel

$\checkmark$ Comparison of bands and estimation of quantity

$\checkmark$ Coupled to densitometry

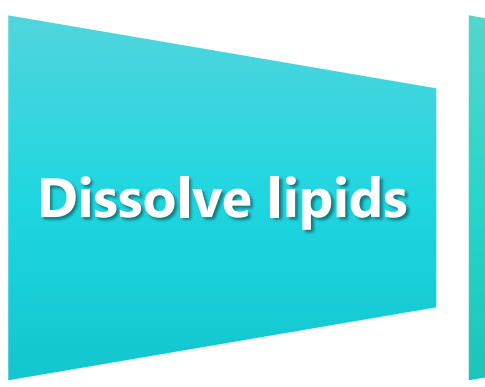

Avoid

precjpitation of CpG or r'tR
Low solvent

interference
Liposomal disruption with binary solvent

Mixture

Chloroform:Methanol

Table 1. Dispersion of CpG or rTR liposomes with mixtures of different chloroform:methanol proportions

\begin{tabular}{|c|c|c|c|}
\hline \multicolumn{2}{|c|}{ MIXTURE } & \multirow{2}{*}{ CpG liposomes } & \multirow{2}{*}{ rTR liposomes } \\
\hline Chloroform & Methanol & & \\
\hline 2 & 1 & Two turbid phases & Two turbid phases \\
\hline 1 & 1 & Two translucent phases & Two phases with suspended particles \\
\hline 1 & 2 & \multirow{2}{*}{ One homogeneous translucent phase } & \multirow{4}{*}{ One phase with suspended particles } \\
\hline 1 & 3 & & \\
\hline 1 & 5 & One phase with suspended particles & \\
\hline \multicolumn{2}{|c|}{ Ethanol } & One turbid phase with suspended particles & \\
\hline
\end{tabular}


Table 2. Determination of the optimal levels of the factors that influence the manufacturing process of rTR liposomes

PROCESS

Lipid film formation by rotary evaporation
RESULTS/FACTORS

Synchronization of parameters such as temperature, time, rotation and pressure is required

\section{SELECTED CONDITIONS}

$20 \mathrm{~min}, 65^{\circ} \mathrm{C}, 180 \mathrm{rpm}$, $800 \rightarrow 400 \rightarrow 70$ mbar

\section{Lipid film dispersion}

Size homogenization by extrusion

Separation by centrifugation

Other important factors
- Sonication produces a high percentage of small undesirable liposomes

- Vortex agitation causes heterogeneous detachment of the film (aggregation of particles)

- Combination of rotation while heating and agitation through vortex generates adequate hydration and homogenous detachment of the film
- Rotation/heating:

$5 \mathrm{~min}, 65^{\circ} \mathrm{C}, 180 \mathrm{rpm}$

- Vortex agitation:

$50 \mathrm{~s}, 1500 \mathrm{rpm}$

11 extrusions through polycarbonate membranes $(1000 \mathrm{~nm})$

Centrifugation time depends on stability (PDI and \%EE) $\quad 6 / 3 \mathrm{~min}, 4^{\circ} \mathrm{C}, 21000 \mathrm{rcf}$

- Lipid amount and proportion

- Hydration medium

- Centrifugation volume
- PC:SA:Ch 7:1,5:4 (30 mg)

- PBS 0,1X (pH 5,8)

- $270 \mu \mathrm{L}$ 
Average PDI: 0,15

A

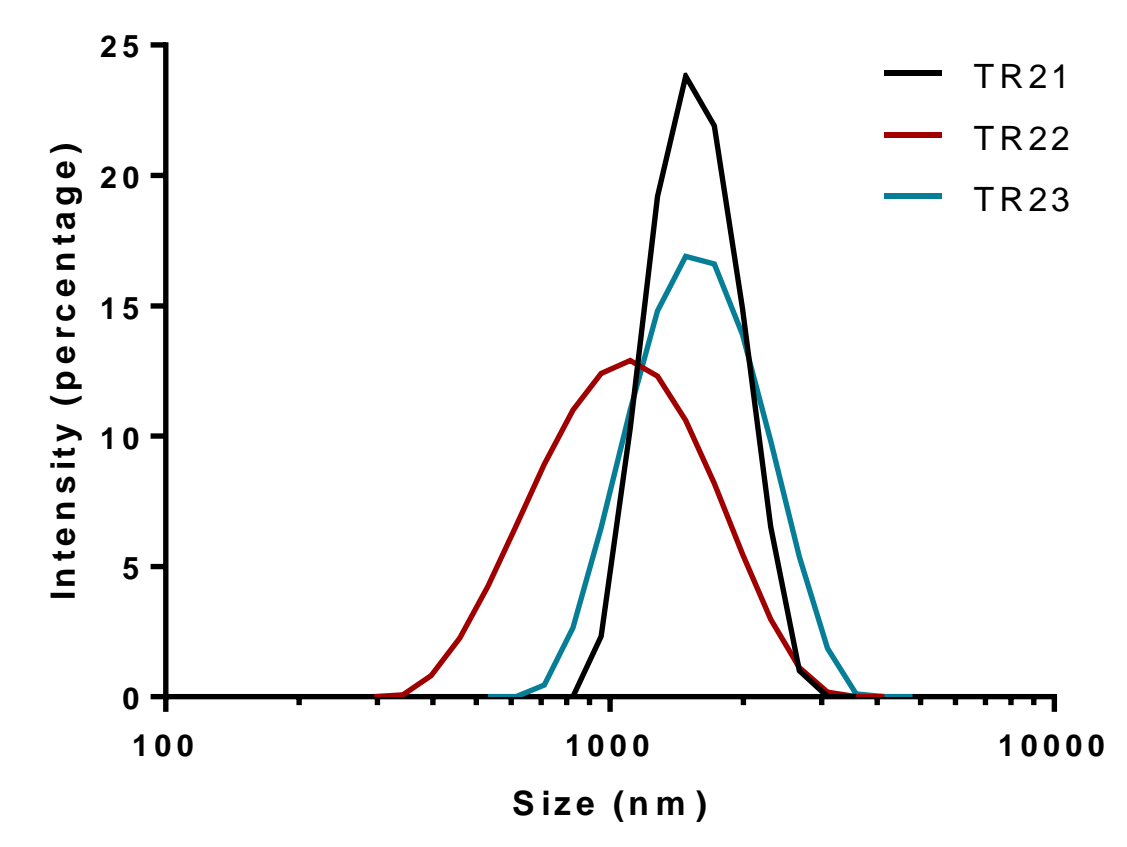

B

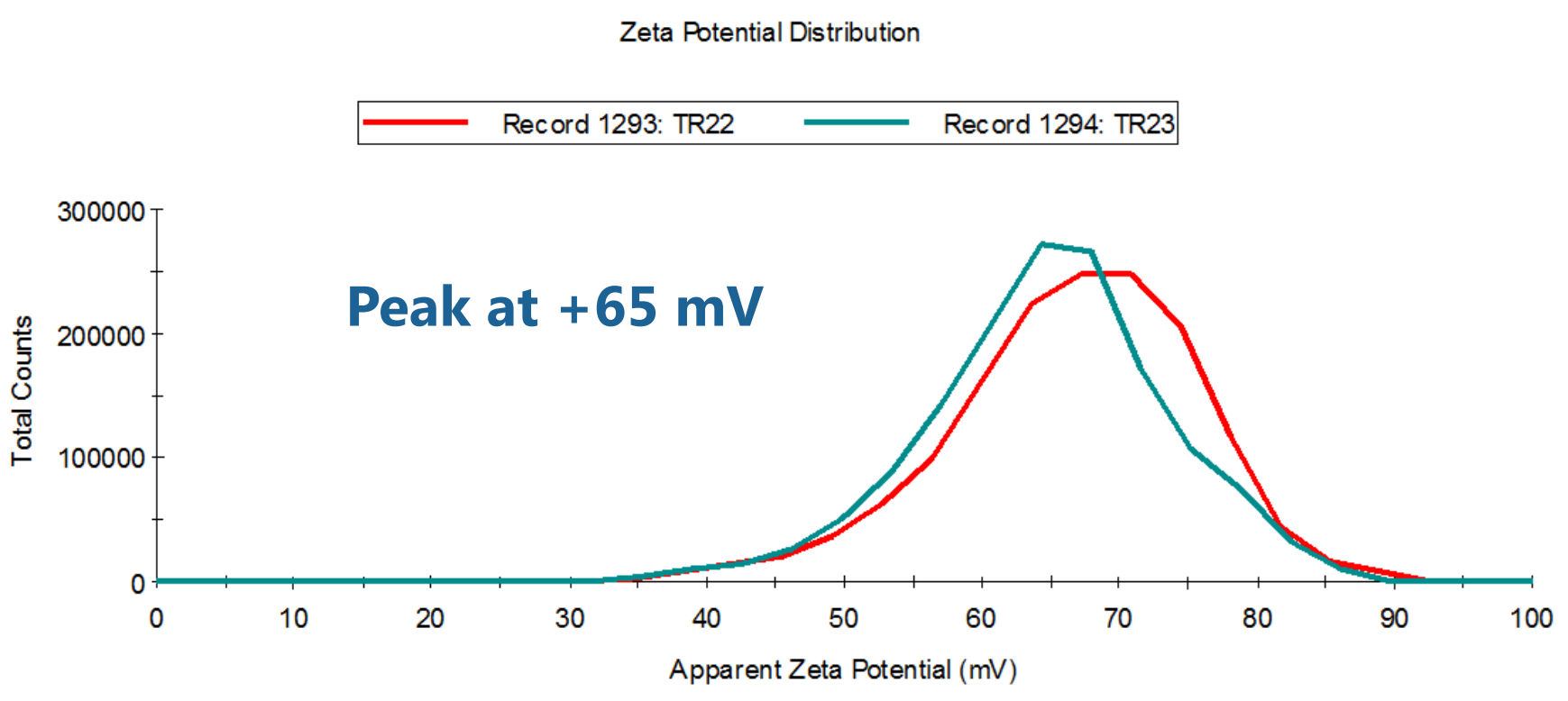

FIGURE 1. rTR liposomes. (A) Size distribution of three batches of $r T R$ liposomes produced under the same conditions. Average PDI: 0.15. (B) Distribution of apparent zeta potential (mV) of two batches of rTR liposomes. A peak at $+65 \mathrm{mV}$ is appreciated in both batches. 


\section{RESULIS rTR quantification}

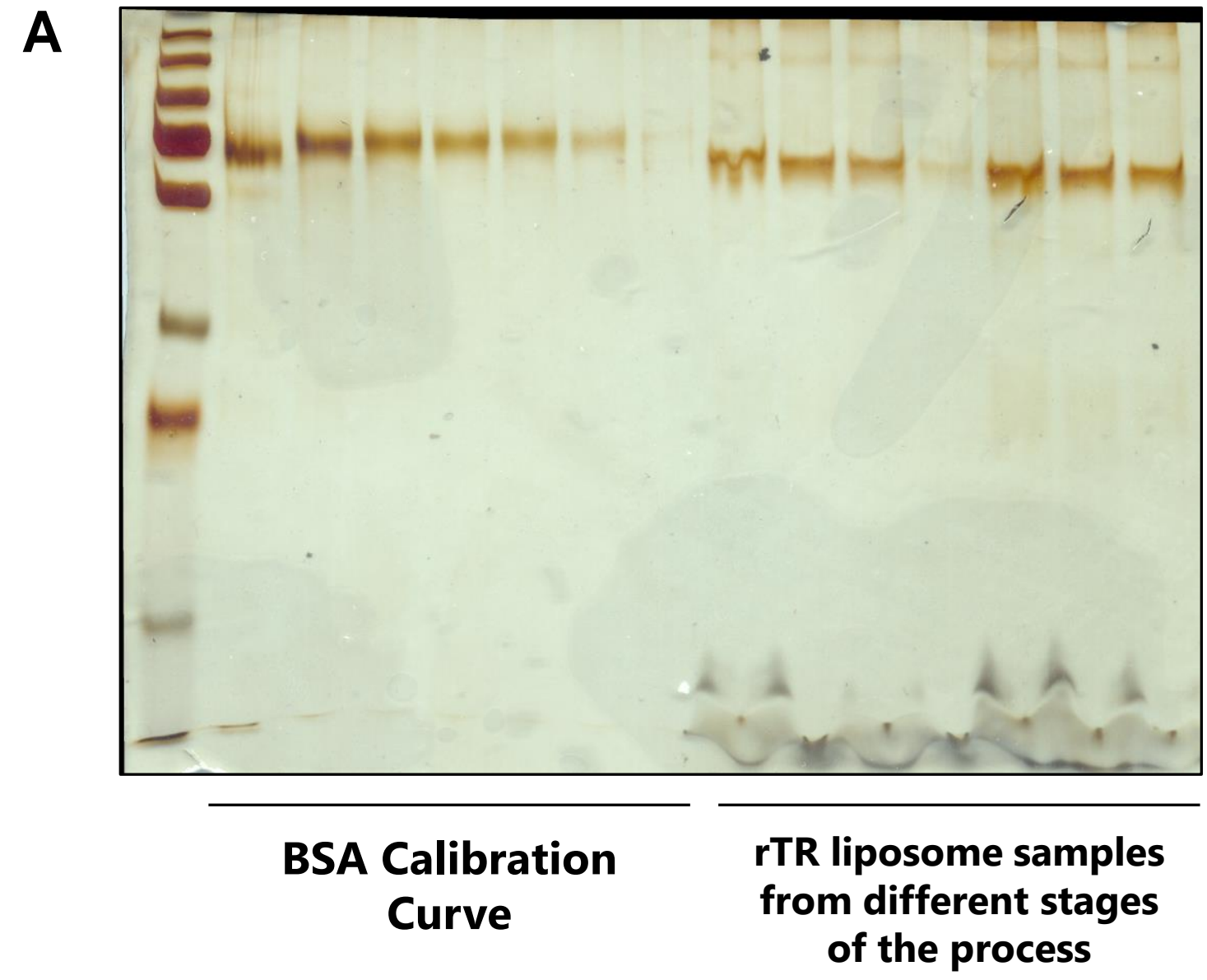

FIGURE 2. rTR quantification. (A) Polyacrylamide gel electrophoresis (SDS-PAGE) of liposomal samples (TR22, different stages of the process) and calibration curve with BSA. In (B) a calibration curve is presented for the quantification of rTR which was obtained from each SDS-PAGE for each analyzed batch.
B

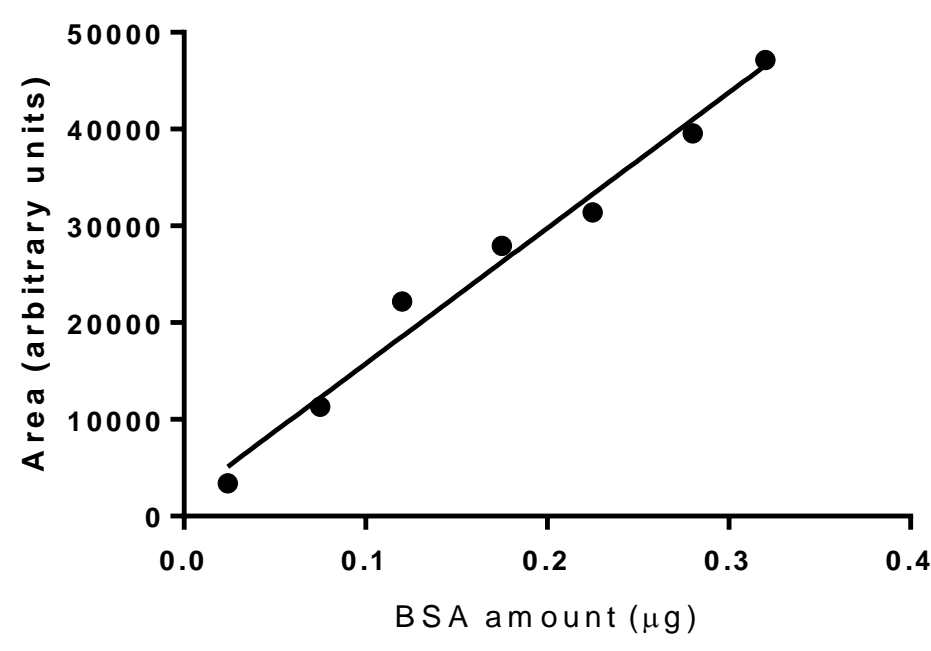

Table 3. Summary of rTR quantification results

\begin{tabular}{|c|c|}
\hline Parameter & STR liposomes \\
\hline $\begin{array}{c}\text { Adjusted amount of analyte in } \\
100 \mu L(\mu g)\end{array}$ & 5.0 \\
\hline $\begin{array}{c}\text { Theoretical encapsulation } \\
\text { efficiency (\%EET) }\end{array}$ & 20.00 \\
\hline $\begin{array}{c}\text { Experimental encapsulation } \\
\text { efficiency (\%EE) }\end{array}$ & 51.20 \\
\hline
\end{tabular}


Table 4. Determination of the optimal levels of the factors that influence the manufacturing process of CpG liposomes

$\begin{array}{ccccccc}\begin{array}{c}\text { Lipid } \\ \text { proportion } \\ (\text { PC:SA:Ch) }\end{array} & \begin{array}{c}\text { Lipid } \\ \text { amount } \\ (\mathrm{mg})\end{array} & \begin{array}{c}\text { Hydration } \\ \text { medium }\end{array} & \begin{array}{c}{[\mathrm{CpG}]} \\ (\mu \mathrm{g} / \mathrm{mL})\end{array} & \begin{array}{c}\text { Dispersion by } \\ \text { rotation/heating }\end{array} & \begin{array}{c}\text { Size homogenization } \\ \text { by extrusion }\end{array} & \begin{array}{c}\text { Separation by } \\ \text { centrifugation }\end{array} \\ 7: 2: 2 & 20,7 & \begin{array}{c}\text { PBS } 0.1 \mathrm{X} \\ \mathrm{pH}=5,8\end{array} & \mathbf{2 0 0} & \begin{array}{c}\mathbf{1 5} \mathbf{m i n}, 65^{\circ} \mathrm{C}, \\ 180 \mathrm{rpm}\end{array} & \begin{array}{c}7 \text { extrusions through } \\ \text { polycarbonate } \\ \text { membranes }(1000 \mathrm{~nm})\end{array} & \begin{array}{c}12 \mathrm{~min}, 4^{\circ} \mathrm{C}, \\ 21000 \mathrm{rcf}\end{array}\end{array}$

The interaction between materials depends on the overcoming of electrostatic repulsion through modulation of temperature, mechanical agitation and progressiveness in the formation of stable vesicles that shield, as they are formed, the CpG negative charge 
We standardized a liposomal formulation which reproducibly encapsulated CpG

Optimal levels were effectively adjusted and reproducible response variables were obtained: Suprananometric size (peak at 900-1000 nm), cationic zeta potential (+65 mV) and physical stability for at least six weeks

A

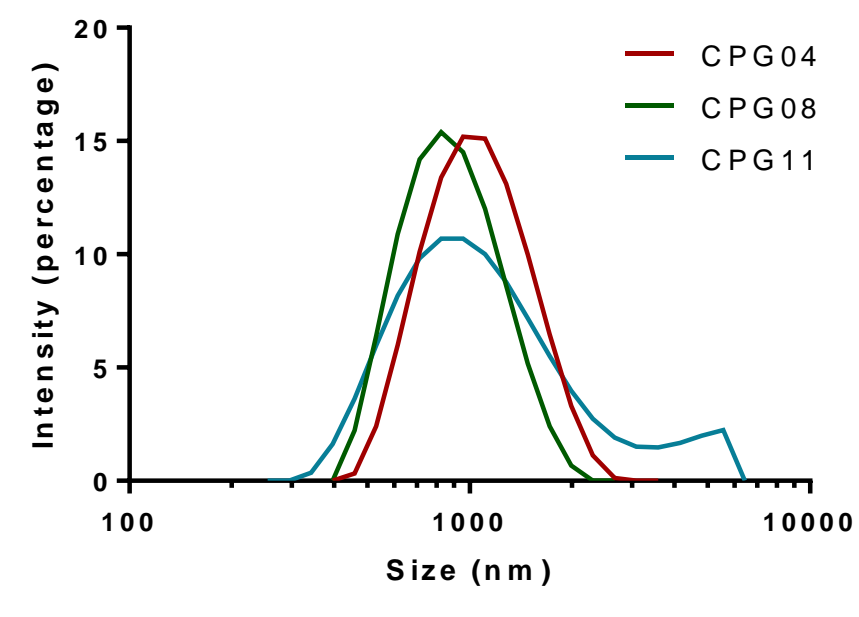

C

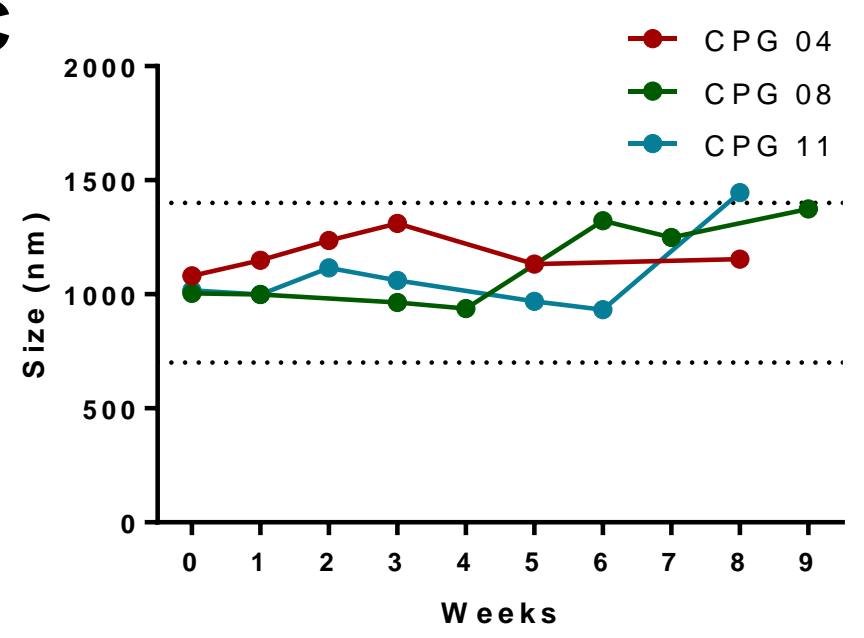

B

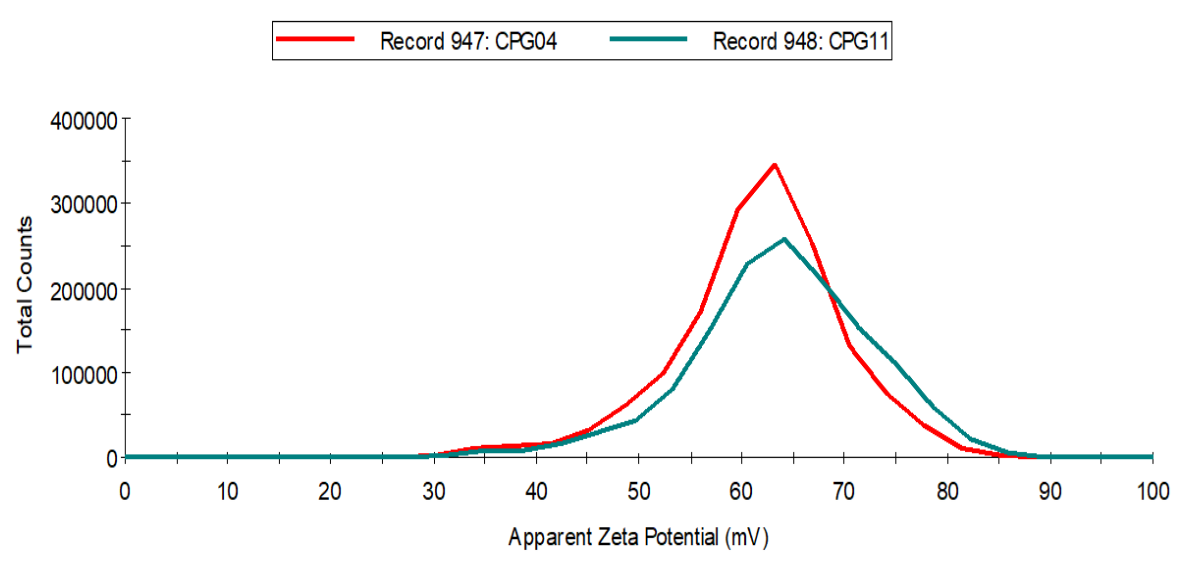

D

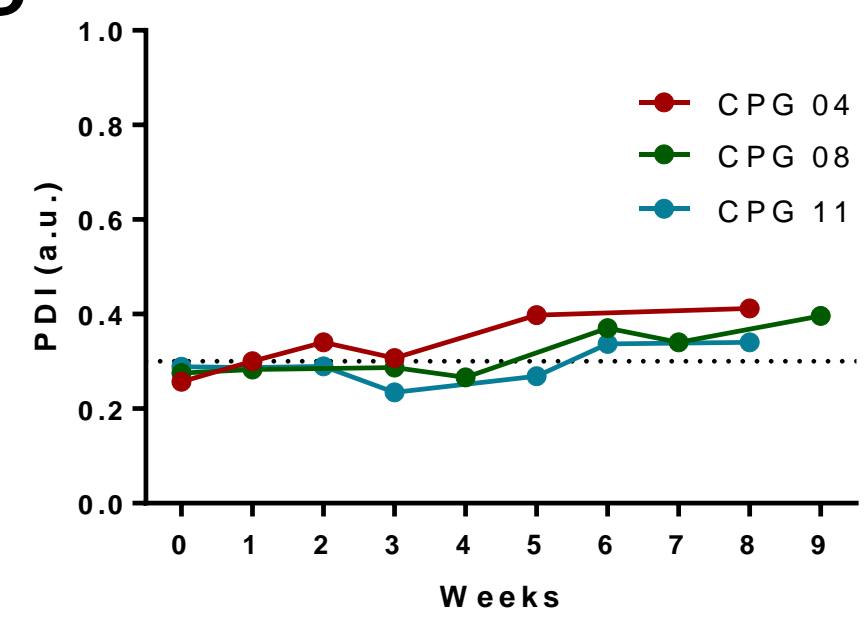

FIGURE 3. CpG liposomes. (A) Size distribution of three batches of $\mathrm{CpG}$ liposomes. (B) Distribution of apparent zeta potential $(\mathrm{mV})$ of two batches of CpG liposomes (CPG04 and CPG11) which present a peak at $+65 \mathrm{mV}$. (C,D) Stability of the CpG liposomes over time (weeks) according to variation of the (C) size (nm) and (D) PDI (a.u.). In (C), it is indicated with dotted horizontal lines the sizes that are considered adequate: between $700 \mathrm{~nm}$ and $1400 \mathrm{~nm}$. In (D), the dotted horizontal line indicates the maximum value of PDI considered acceptable: 0.3 . PDI: polydispersity index, a.u.: arbitrary unit. 


\section{RESULTS CPG quantification}

A

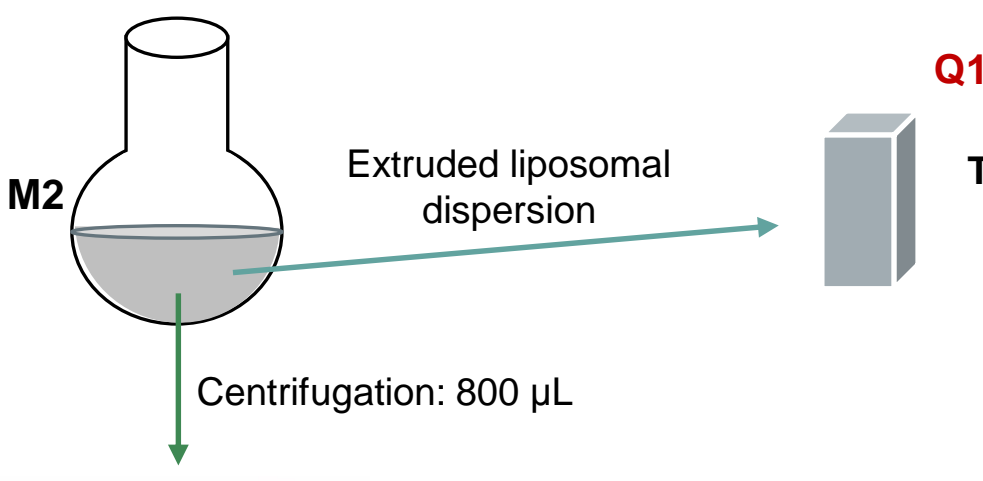

Q1 $\rightarrow 50 \mu \mathrm{L}$ aliquot

Total volume: $600 \mu \mathrm{L}$

Spectrophotometer

measurement
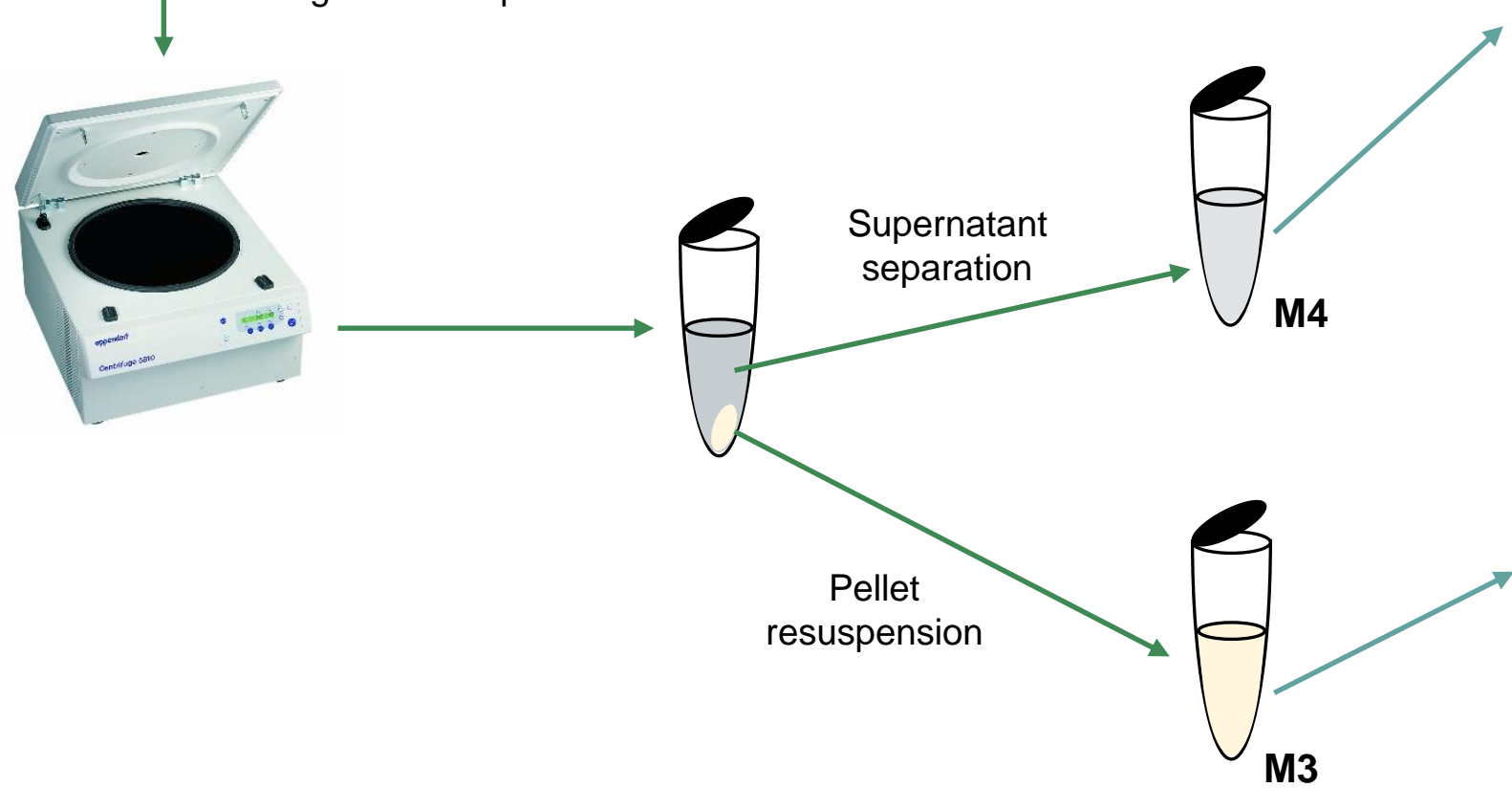

Final volumen: $800 \mu \mathrm{L}$

FIGURE 4. CPG quantification. (A) Schematic representation of the sampling system for the direct quantification of $\mathrm{CpG}$ in several stages of the manufacturing process of liposomes. In (B) a calibration curve is presented for the quantification of $\mathrm{CpG}$.

\section{Direct quantification}

Q2 $\rightarrow 100 \mu \mathrm{L}$ aliquot

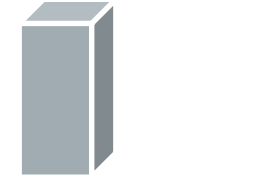

Total volume: $600 \mu \mathrm{L}$ Spectrophotometer measurement

Q3 $\rightarrow 50 \mu \mathrm{L}$ aliquot

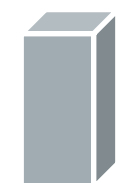

Total volume: $600 \mu \mathrm{L}$ Spectrophotometer measurement
Evaluation of the reliability of the method

\section{Calculations}

regarding theoretical and experimental values

B

Calibration Curve CpG in chloroform:methanol 1:2

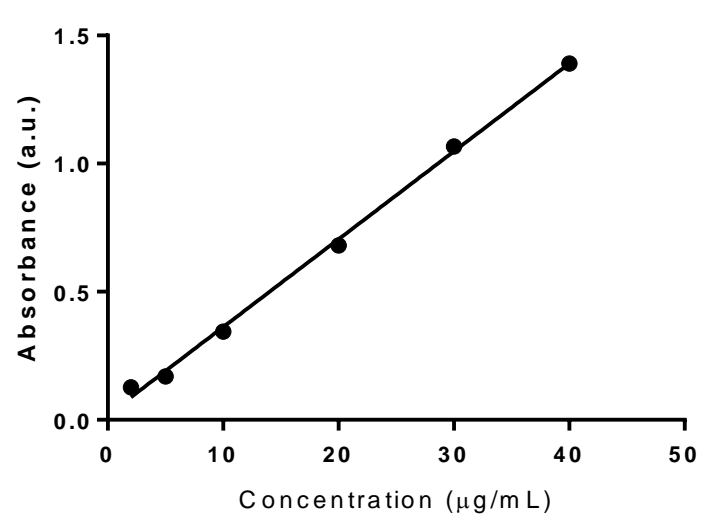


Table 5. Summary of CpG quantification results

\begin{tabular}{cc}
\hline Parameter & Mean of calculated values $(n=8)$ \\
\hline $\begin{array}{c}\text { Percentage of CpG in } \\
\text { resuspended pellet (\%) }\end{array}$ & 89 \\
$\begin{array}{c}\text { Percentage of CpG in } \\
\text { dispersion (\%) }\end{array}$ & 94 \\
$\begin{array}{c}\text { Theoretical encapsulation } \\
\text { efficiency (\%EET) }\end{array}$ \\
$\begin{array}{c}\text { Experimental } \\
\text { encapsulation efficiency } \\
\text { (\%EEE) }\end{array}$ & 64.53 \\
\hline
\end{tabular}




\section{Main effects that influence liposome preparation}
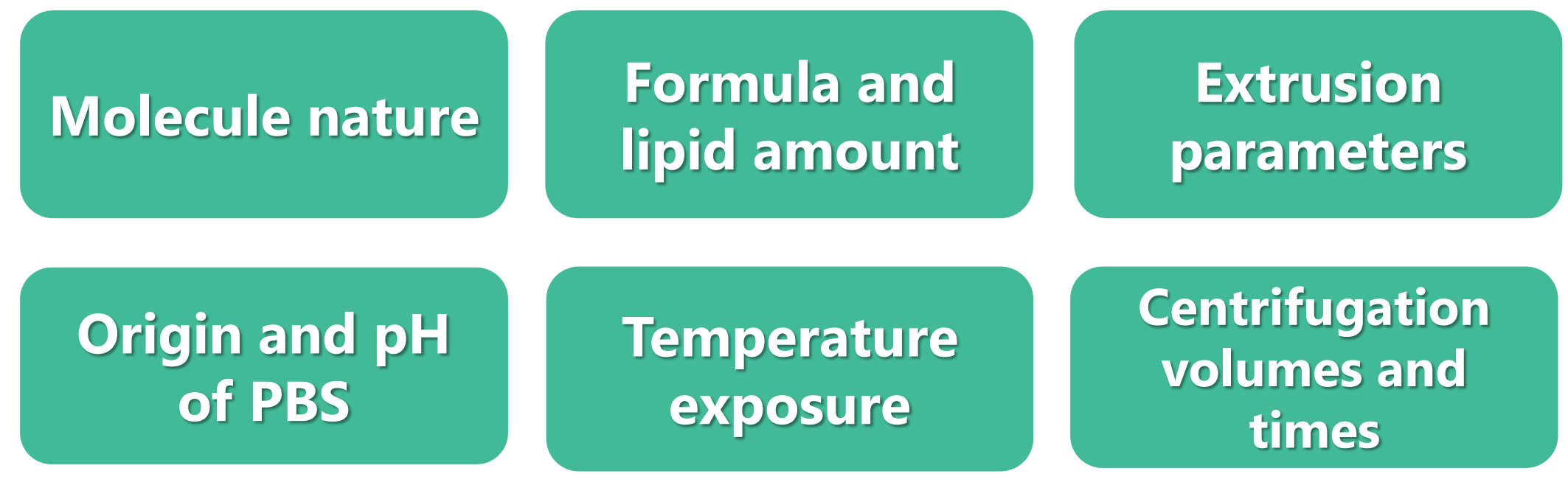


\section{RESULTS Employed liposomes for in vivo experiment}

A

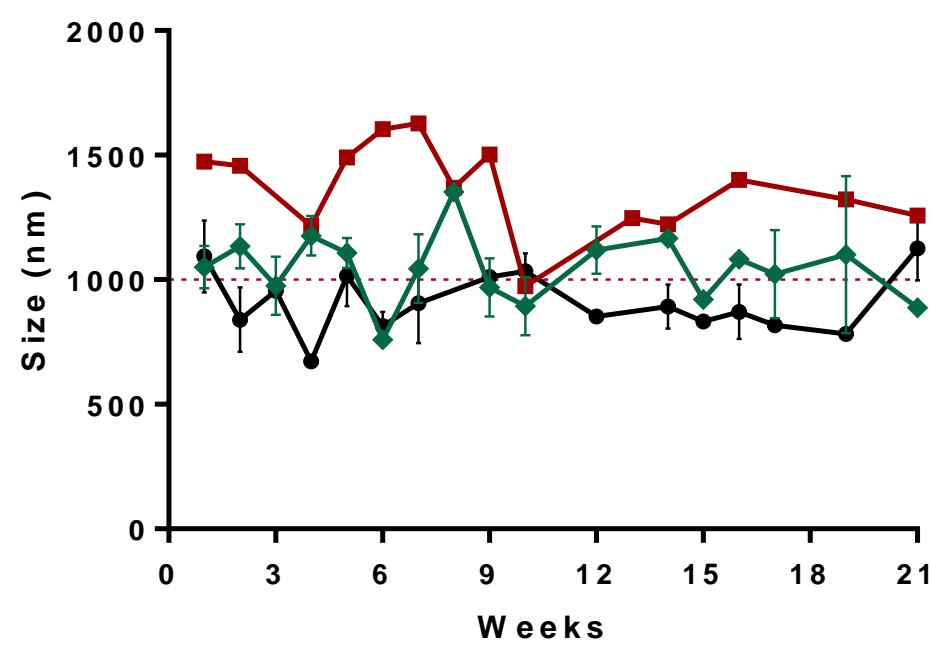

B

$\rightarrow(C p G)$

$\rightarrow(r T R)$

$\rightarrow(\mathrm{Chol})$

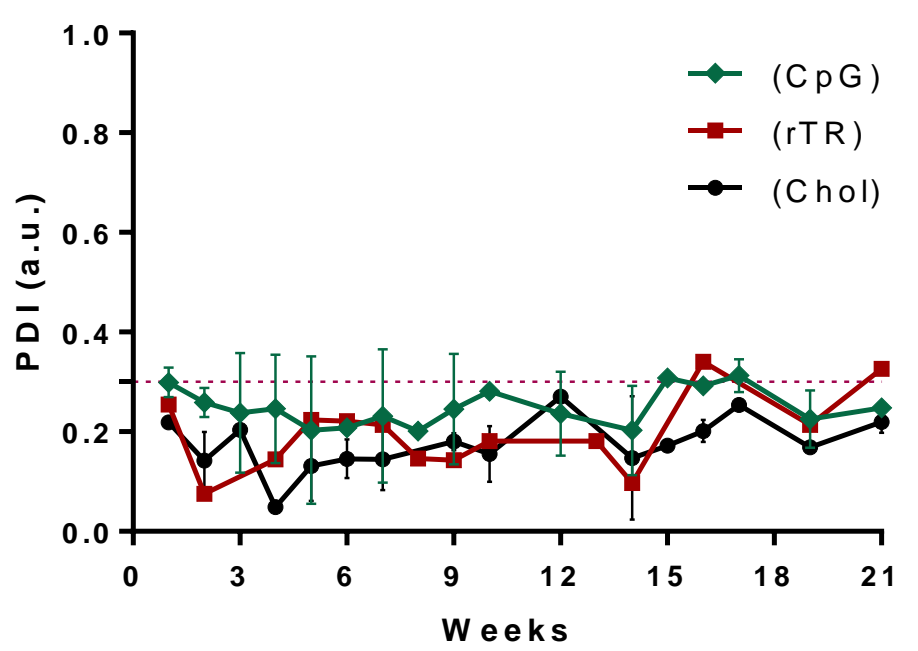

C

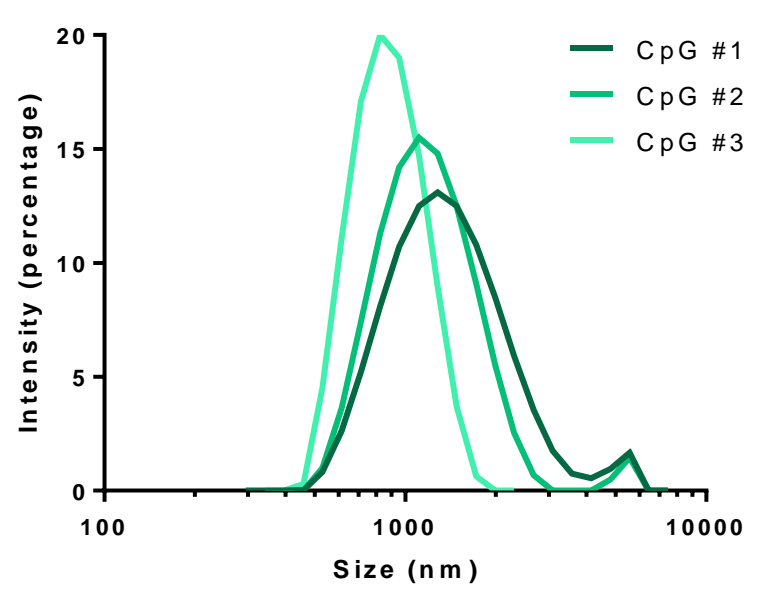

D

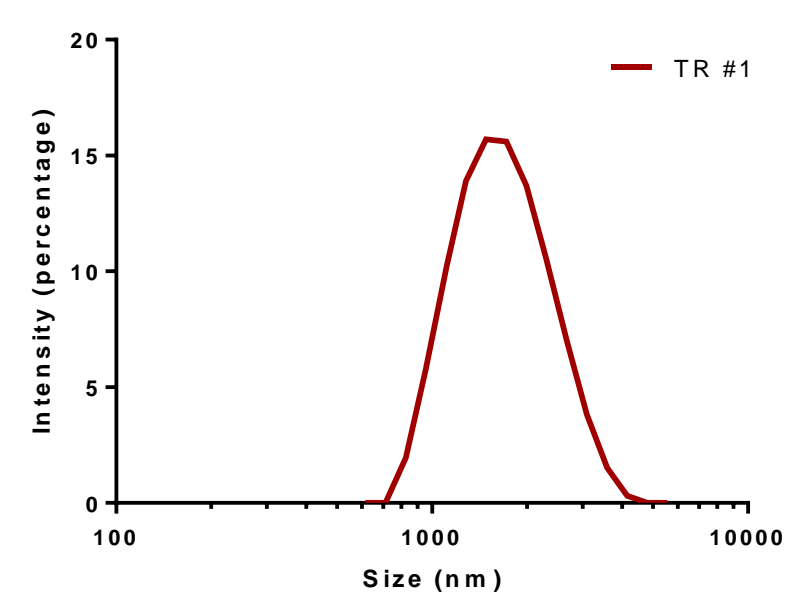

E

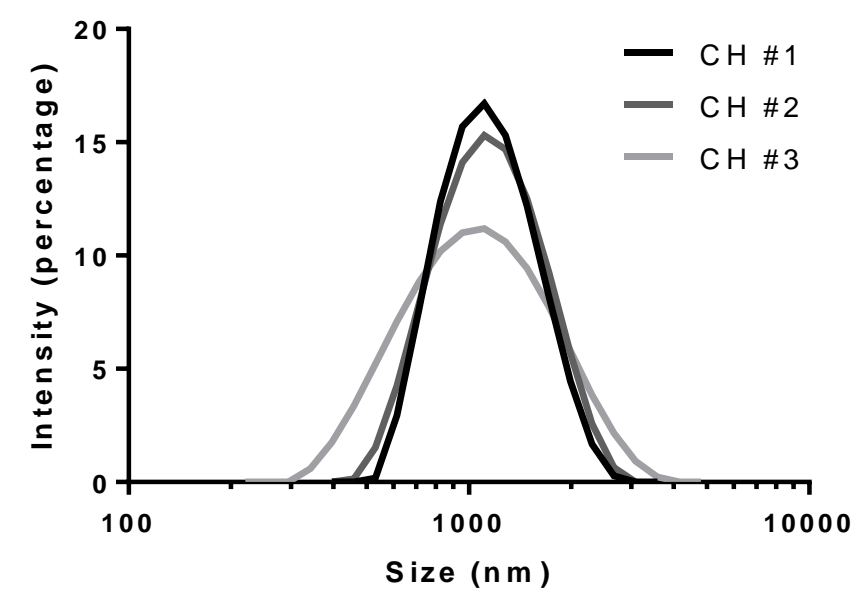

FIGURE 5. Employed liposomes for in vivo experiment. (A-B) Stability of the employed liposomes for in vivo experiment, over time (21 weeks) according to variation of the (A) size (nm) and (B) PDI (a.u.). In (A), it is indicated with a dotted horizontal line the $1000 \mathrm{~nm}$ size as guidance. In (B), the dotted horizontal line indicates the maximum value of PDI considered acceptable: 0.3. (C-E) Size distribution of the batches of liposomes produced under the same conditions, (C) CpG liposomes, (D) rTR liposomes, (E) PBS liposomes -negative control-. PDI: polydispersity index, a.u.: arbitrary unit. 
Experimental groups of treated mice ()$=$ liposomal; example: $(C p G)=C p G$ encapsulated in liposomes Dosages: $r$ TR $\rightarrow 5 \mu \mathrm{g}, \mathrm{CpG} \rightarrow 12,5 \mu \mathrm{g}$
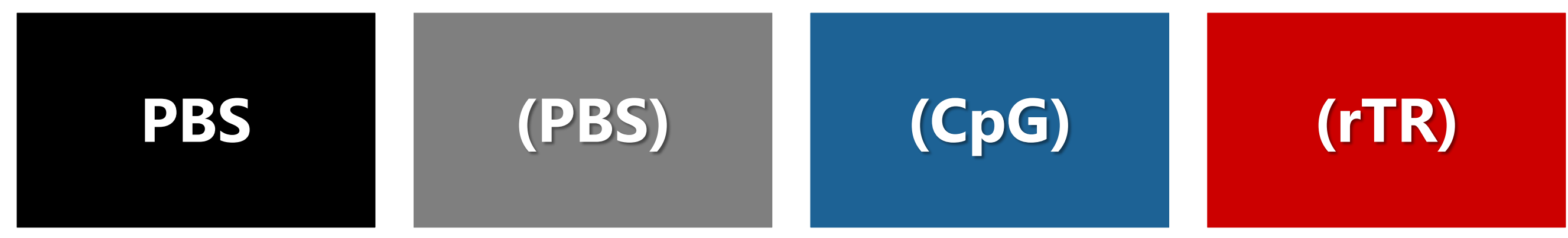

$$
\begin{gathered}
(r T R)+ \\
\text { CPG }
\end{gathered}
$$

rTR +

(CpG)

$$
\begin{gathered}
(r T R)+ \\
(C p G)
\end{gathered}
$$


A

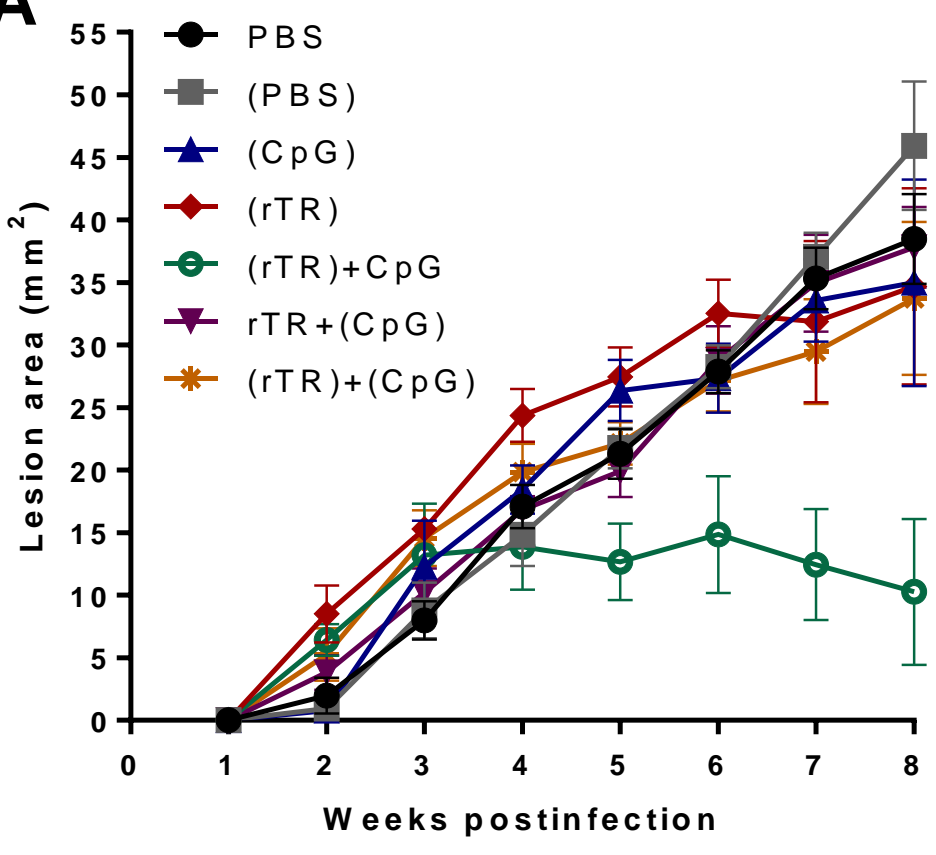

C

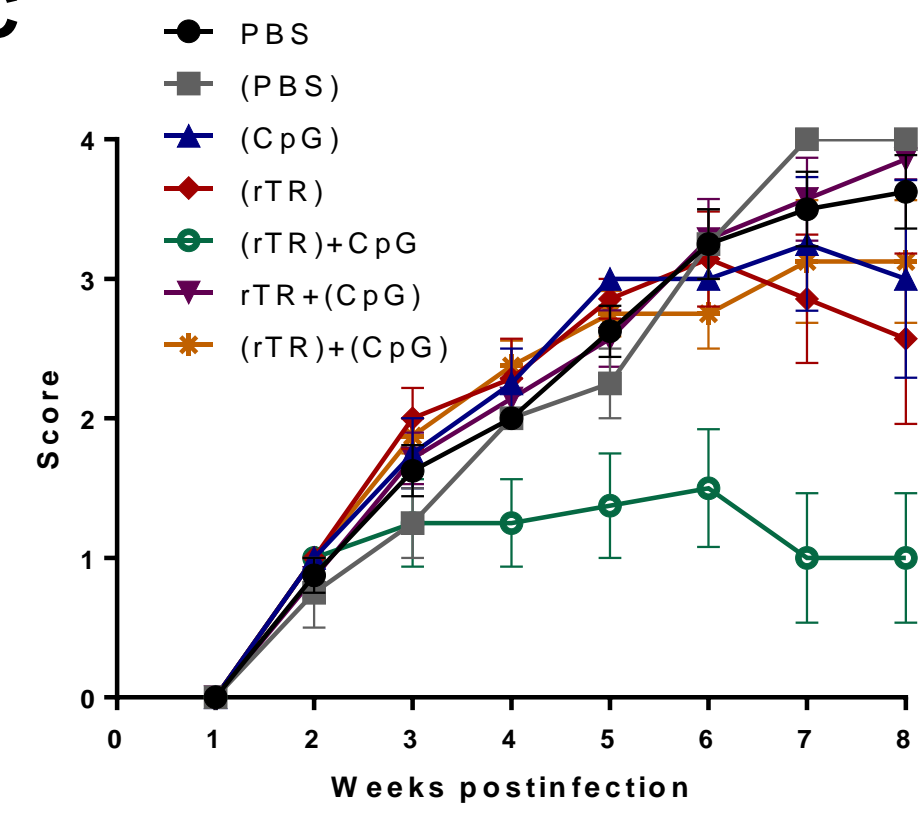

B

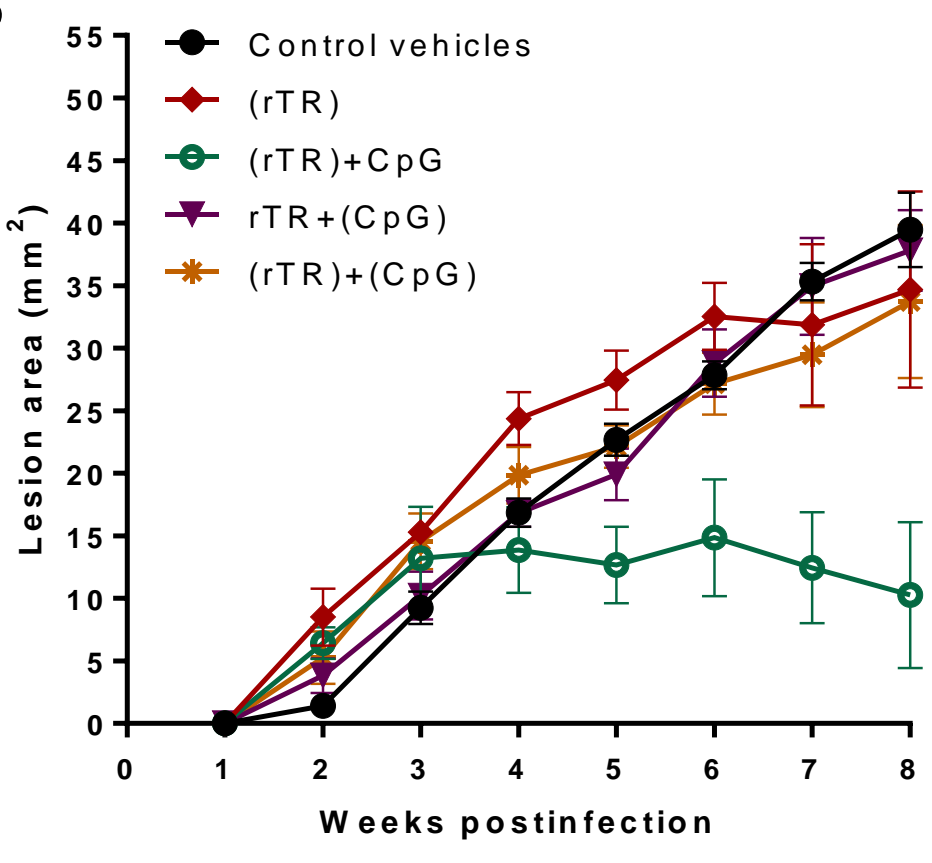

D

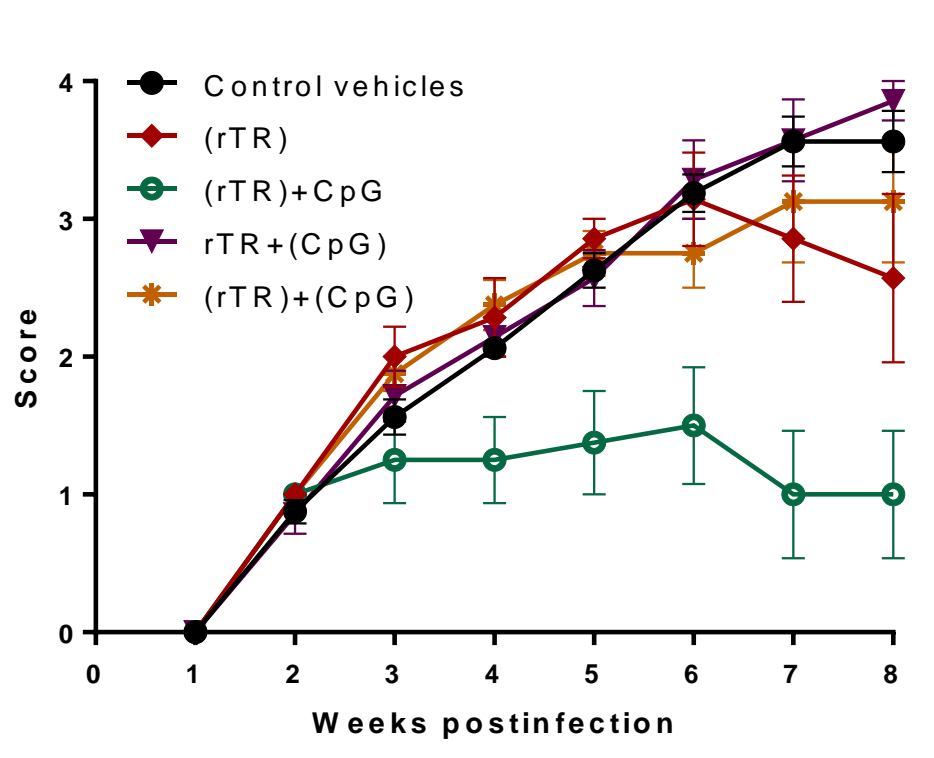

FIGURE 6. Lesion area kinetics and score measurements, clinical followup, from the in vivo experiment. $\mathrm{BALB} / \mathrm{C}$ mice were infected in the dermis of the ear and then it was performed the clinical follow-up in terms of (A-B) lesion area and (C-D) score. In (B,D) negative control mice were pooled: PBS, (PBS) and (CpG). 
A

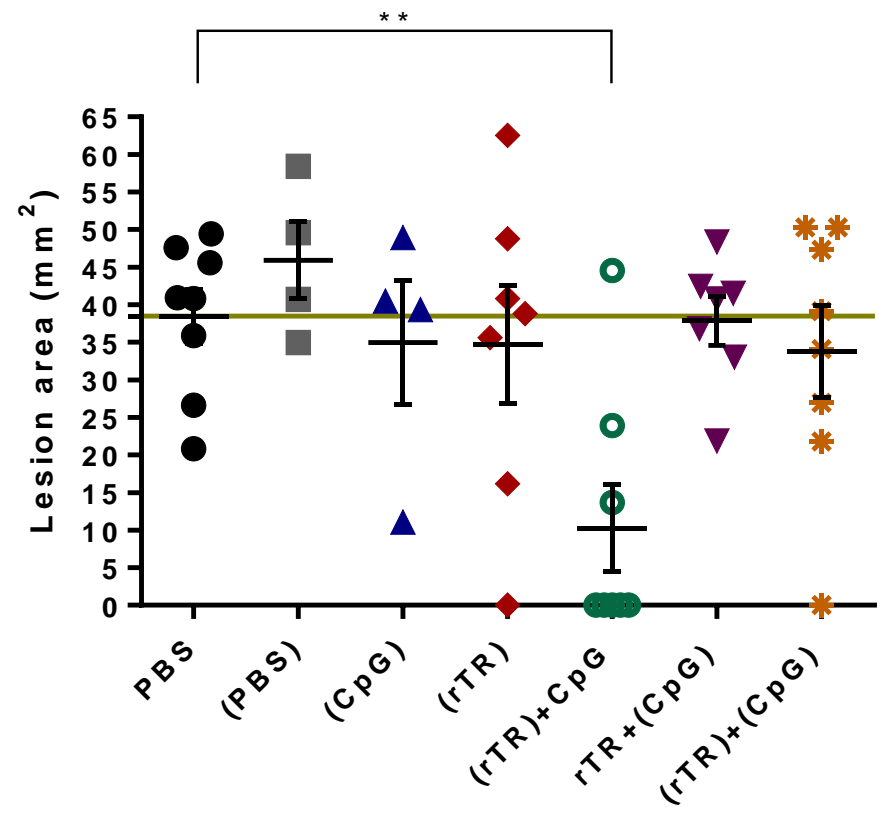

C

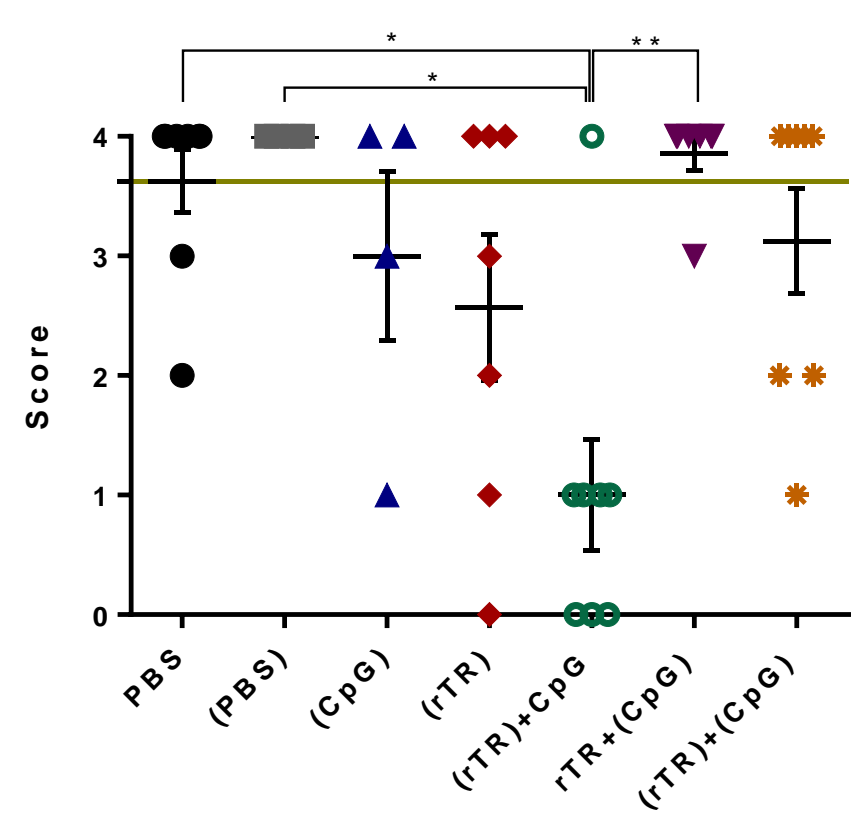

B

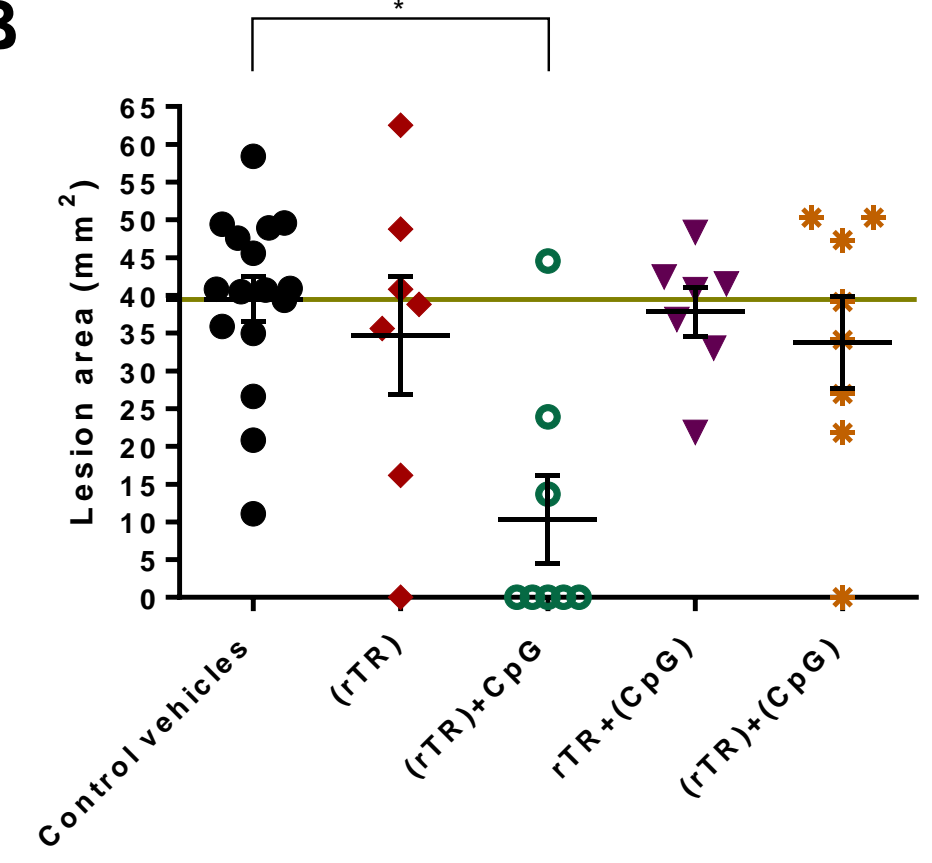

D

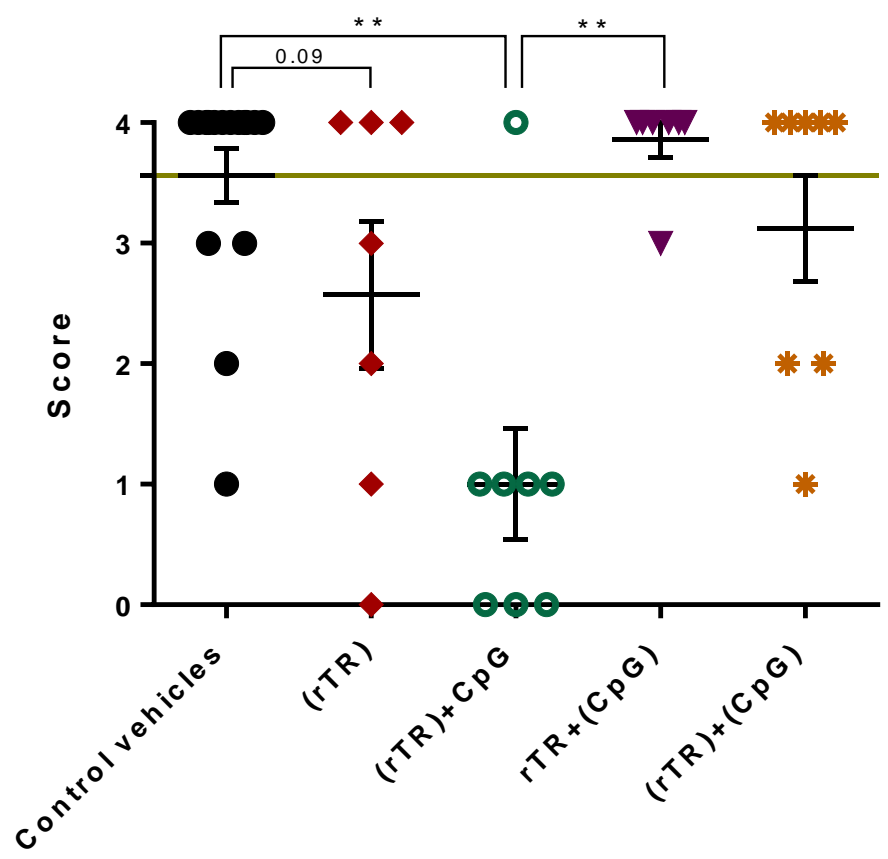

FIGURE 7. Lesion area and score of the mice in week 8 post-infection. In week 8 post-infection, clinical measurements from each mice are shown in terms of (A-B) lesion area and (C-D) score. In (B,D) negative control mice were pooled: PBS, (PBS) and (CPG). Statistic analysis was performed as follows: (A) Unpaired Mann-Whitney non-parametric $t$-test. (B-C) One-way ANOVA (Kruskal-Wallis nonparametric test) with Dunn's multiple comparisons test. In (D), the statistical analysis between control vehicles or $r T R+(C p G)$ and $(r T R)+C p G$ was performed using the same approach as (B-C). Also, in (D), the $P<0,09$ between control vehicles and (rTR) was obtained as explained for (A). $P<0,05$ in any case except for comparison between control vehicles and (rTR) in (D). The olive green horizontal line for each graph is plotted to show the mean values of the PBS or control vehicles groups. 


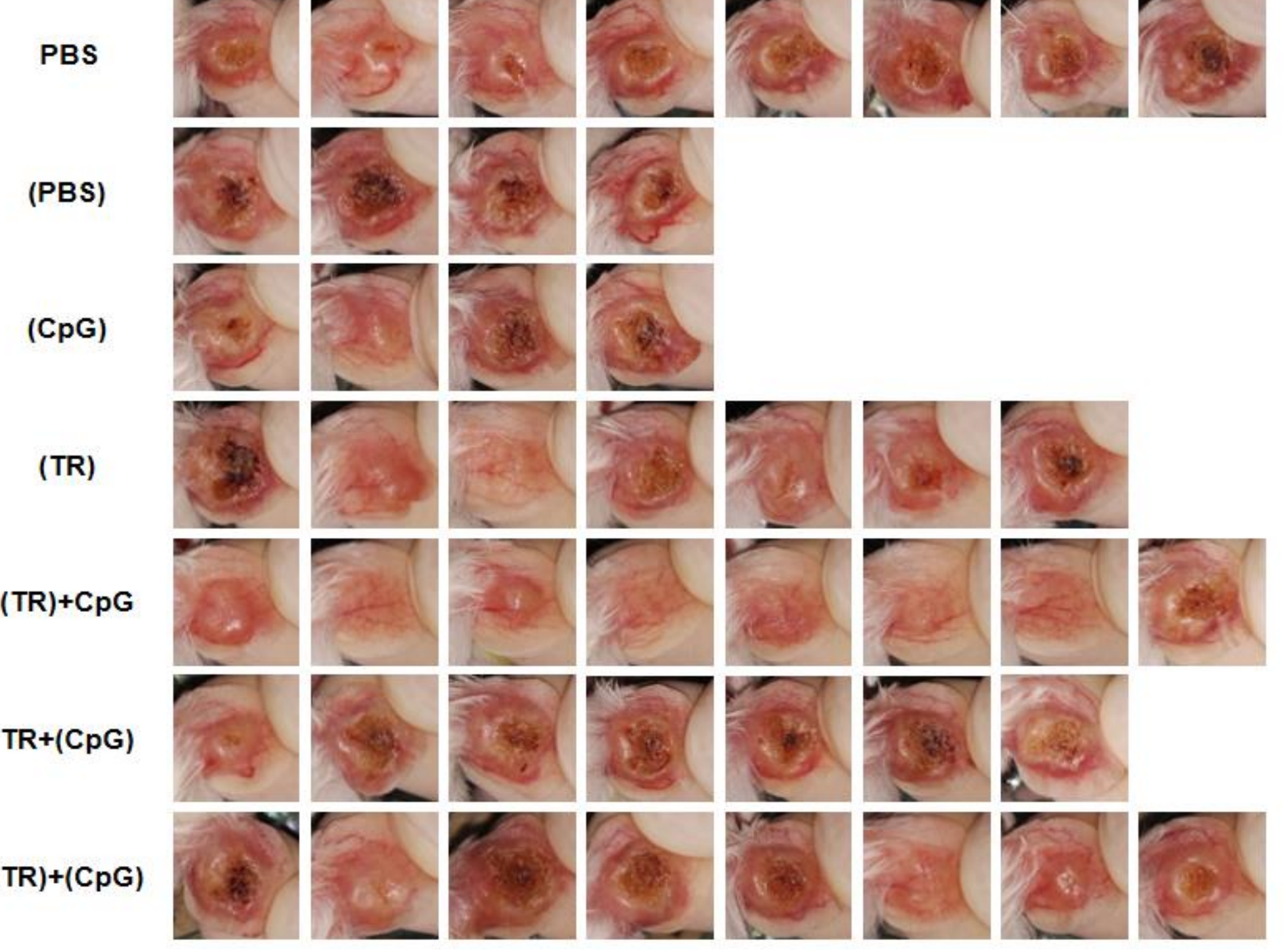

FIGURE 8. Clinical appearance of the lesions shown with photographs of the infected ears for each mice, in each group, in week 8 post-infection. 
A

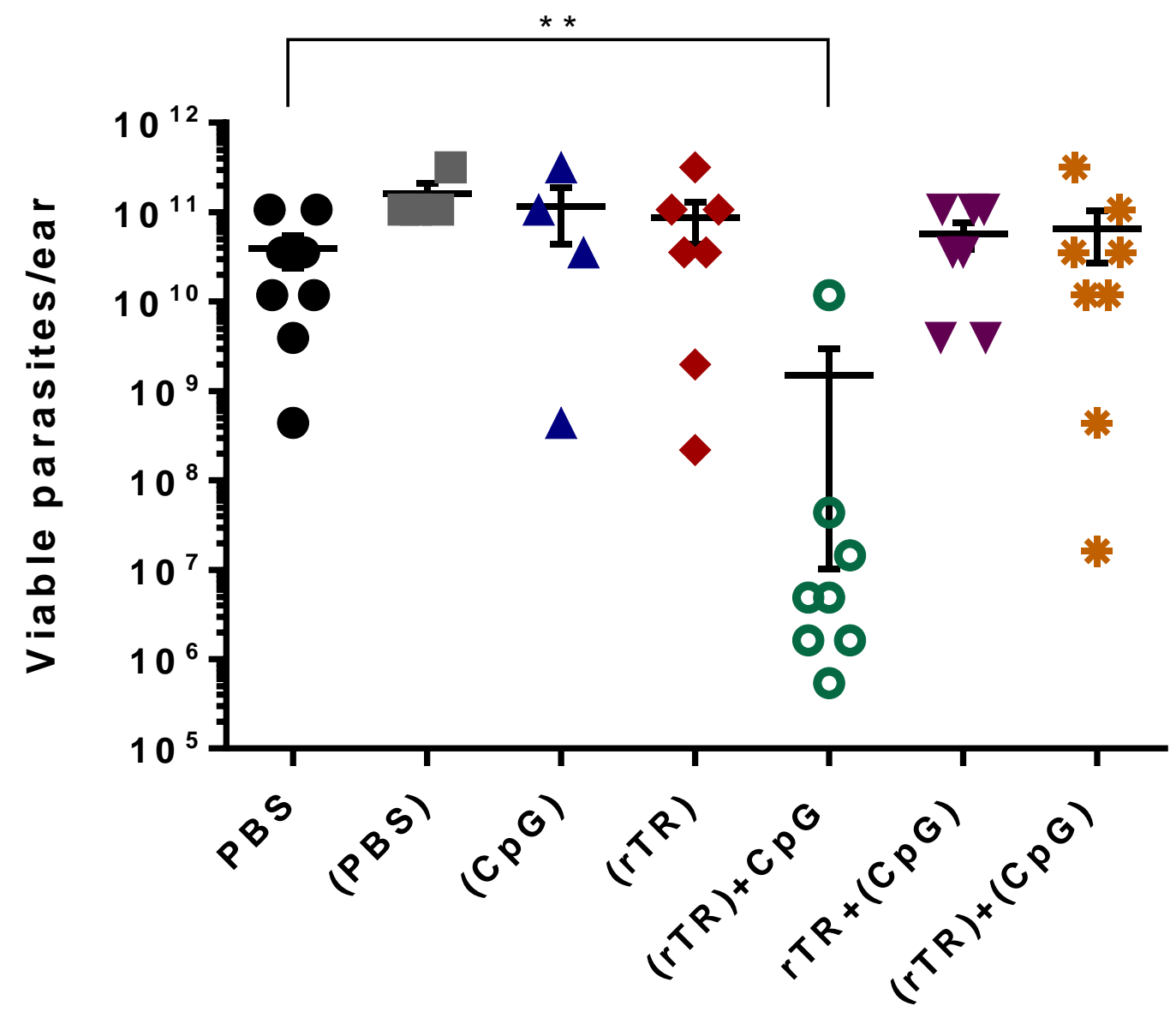

B

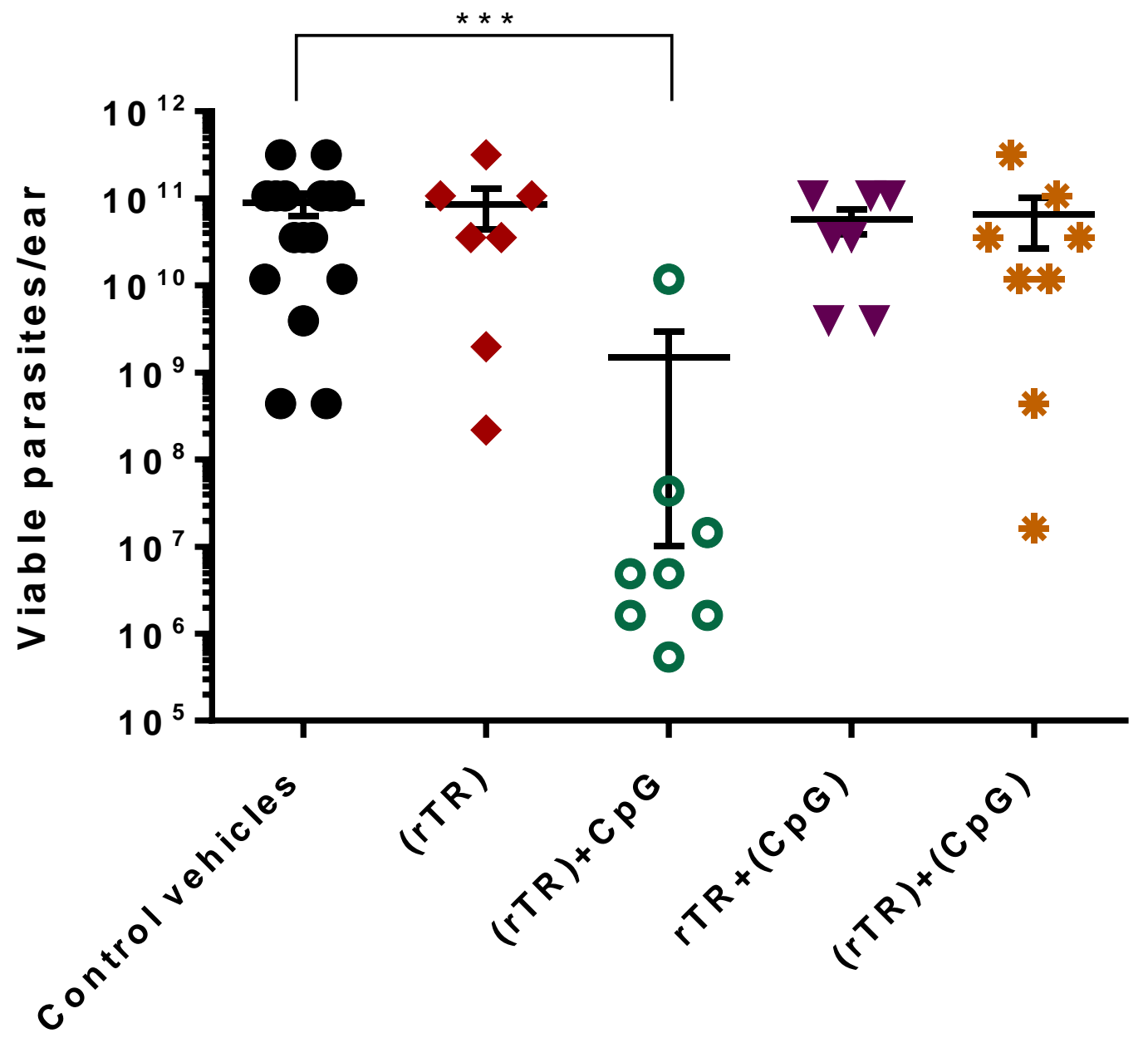

FIGURE 9. Parasitic loads from the in vivo experiment. Viable parasites per ear for each mice group with (A) individual negative controls or (B) pooled negative controls [control vehicles] - PBS, (PBS) and (CpG). Statistic analysis was performed as follows: (A) Unpaired Mann-Whitney non-parametric $t$-test. (B) One-way ANOVA (Kruskal-Wallis nonparametric test) with Dunn's multiple comparisons. $\mathrm{P}<0,05$ in any case. 
A

$\lg G 1$

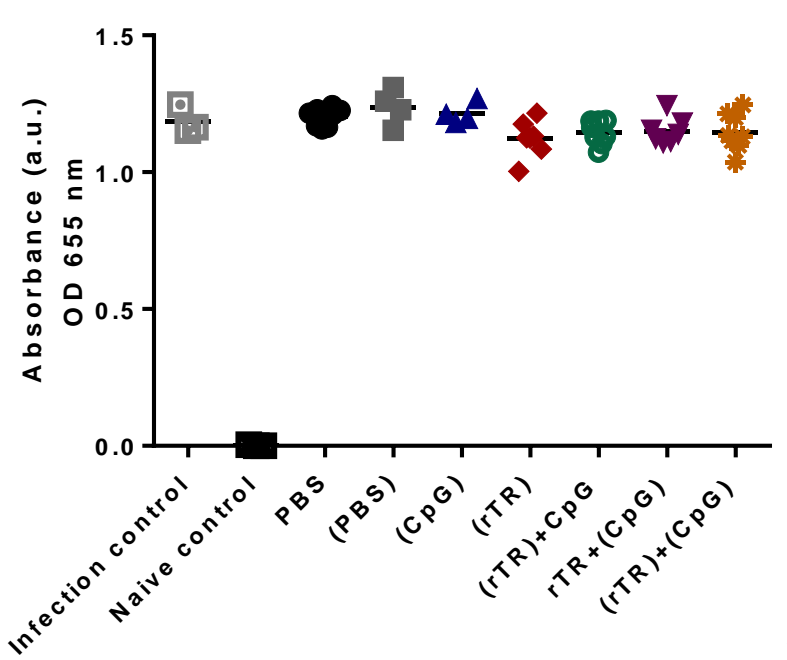

D

$\lg G 1$

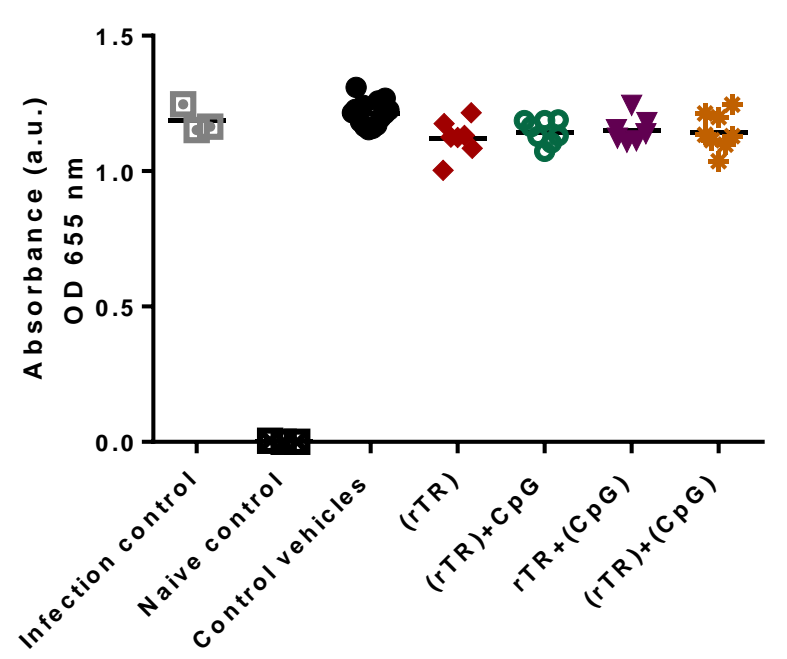

B

$\lg G 2$ a
$E$
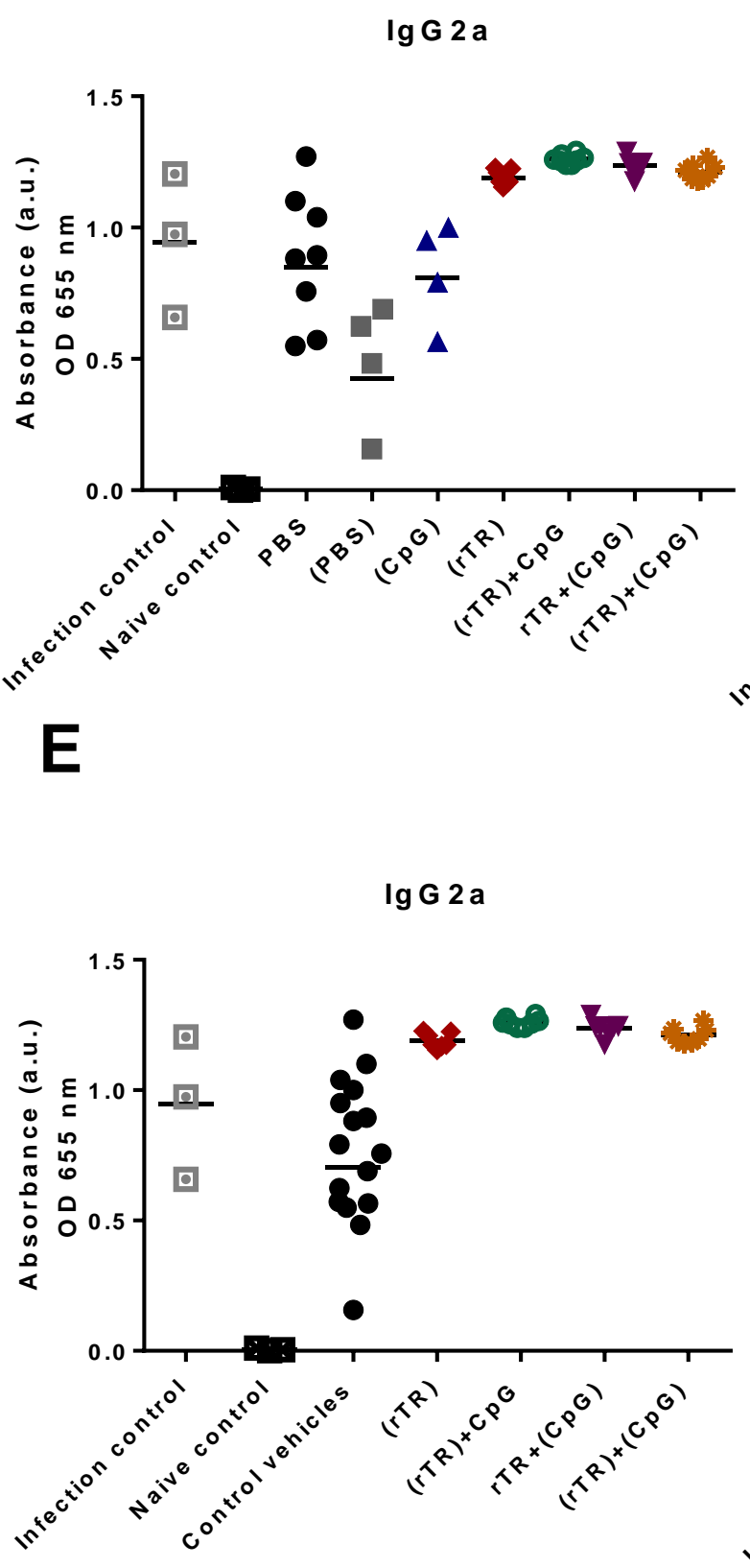

C

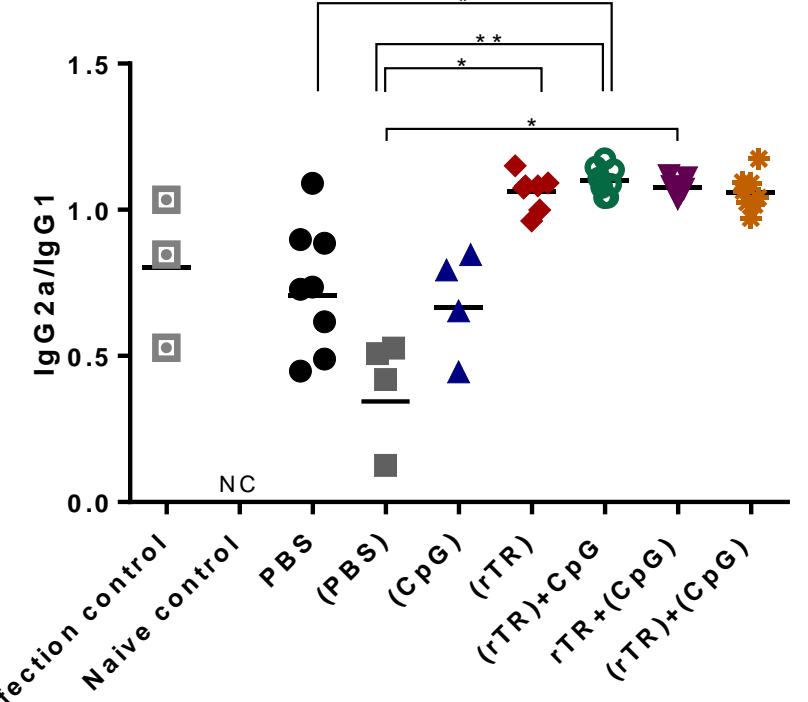

$F$

$\lg G 2 a / \lg G 1$

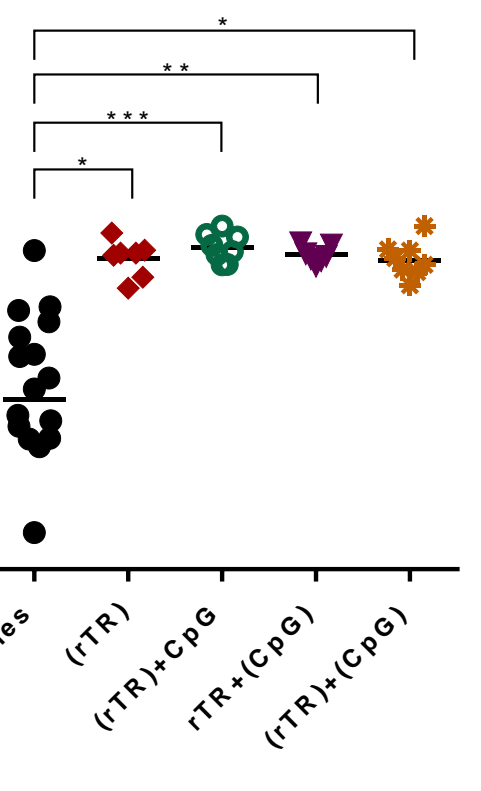

FIGURE 10. Serum levels of IgG1 and IgG2a type antibodies. (A,B,D,E) Absorbance (a.u.) or optical density (OD) at $655 \mathrm{~nm}$ of the chromogenic reaction to determine anti-rTR (A,D) IgG1 or (B,E) IgG2a antibodies. (C,F) calculated IgG2a/IgG1 ratio from the measurement of the anti-rTR antibody levels determined in the sera of the mice after euthanasia (8 weeks post-infection) [shown in (A,B, D, E)]. (A-C) Individual negative controls or (D-F) pooled negative controls [control vehicles] - PBS, (PBS) and (CpG). Statistic analysis was performed as follows: one-way ANOVA (Kruskal-Wallis non-parametric test) with Dunn's multiple comparisons. $\mathrm{P}<0,05$ in any case. a.u.: arbitrary units. 


\section{CONCLUSIONS}

Cationic liposomes with homogeneous suprananometric size were prepared with high CPG encapsulation efficiency

Encapsulation efficiency was $~ 70 \%$ for CpG liposomes, and $\sim 20 \%$ for rTR liposomes, and the procedure was reproducible enough to perform an in vivo experiment
Preparation of micrometric cationic vesicles that encapsulate rTR protein was standardized without degrading nor aggregating it

$87,5 \%[7 / 8]$ of the mice vaccinated with $(r T R)+C p G$ were protected against the infectious challenge, with lower parasitic load and higher IgG2a/IgG1 antibody ratio vs. control vehicles 


\section{PERSPECTIVES}

\section{Co-encapsulation of CpG and rTR}

Optimization the scheme and dosage of the vaccine to increase effectiveness

Advanced characterization of the most effective liposome formulation 


\section{ACKNOWLEDGMENTS AND FUNDING}

"Colloids" Research Group for their technical support regarding physical characterization

"Biopolimer" Research Group for providing us access to their facilities and equipment

\section{COLCIENCIAS}

Project 1115-519-29213

Project 1115-569-33796

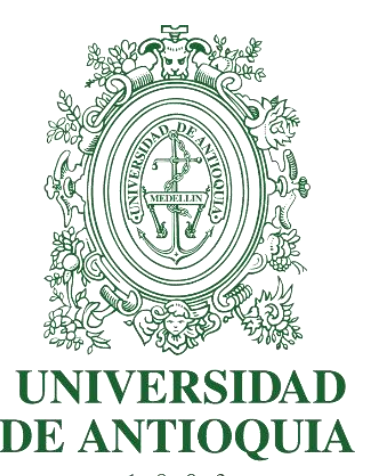

Committee for the Development of Research of the University of Antioquia -CODI-

Project CPT-1217 


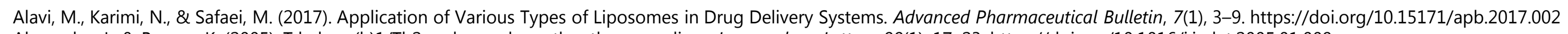
Alexander, J., \& Bryson, K. (2005). T helper (h)1/Th2 and: paradox rather than paradigm. Immunology Letters, 99(1), 17-23. https://doi.org/10.1016/j.imlet.2005.01.009

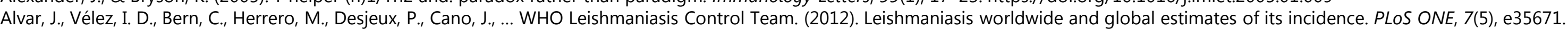
https://doi.org/10.1371/journal.pone.0035671

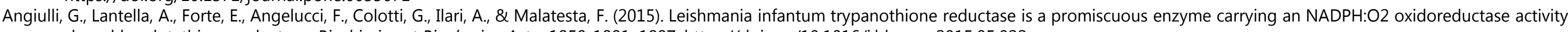
shared by glutathione reductase. Biochimica et Biophysica Acta, 1850, 1891-1897. https://doi.org/10.1016/j.bbagen.2015.05.022

Askarizadeh, A., Jaafari, M. R., Khamesipour, A., \& Badiee, A. (2017). Liposomal adjuvant development for leishmaniasis vaccines. Therapeutic Advances in Vaccines, 5(4-5), 85-101. https://doi.org/10.1177/2051013617741578

Badiee, A., Heravi Shargh, V., Khamesipour, A., \& Jaafari, M. R. (2013). Micro/nanoparticle adjuvants for antileishmanial vaccines: Present and future trends. Vaccine, 31(5), 735-749. https://doi.org/10.1016/j.vaccine.2012.11.068

Bode, C., Zhao, G., Steinhagen, F., Kinjo, T., \& Klinman, D. M. (2011). CpG DNA as a vaccine adjuvant. Expert Review of Vaccines, 10(4), 499-511. https://doi.org/10.1586/erv.10.174

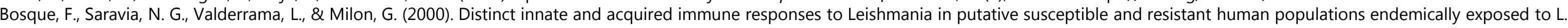
(Viannia) panamensis infection. Scandinavian Journal of Immunology, 51, 533-541. https://doi.org/10.1046/j.1365-3083.2000.00724.x

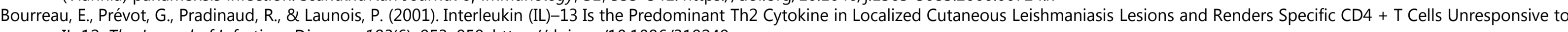
IL-12. The Journal of Infectious Diseases, 183(6), 953-959. https://doi.org/10.1086/319249

Bramwell, V. W., \& Perrie, Y. (2005). The rational design of vaccines. Drug Discovery Today, 10(22), 1527-1534. https://doi.org/10.1016/S1359-6446(05)03600-7

Chakravarty, J., \& Sundar, S. (2010). Drug resistance in leishmaniasis. Journal of Global Infectious Diseases, 2(2), 167. https://doi.org/10.4103/0974-777X.62887

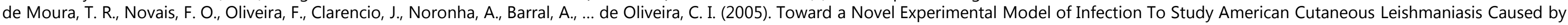
Leishmania braziliensis. Infection and Immunity, 73(9), 5827-5834. https://doi.org/10.1128/IAI.73.9.5827-5834.2005

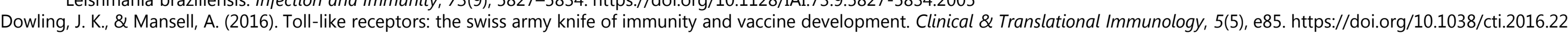

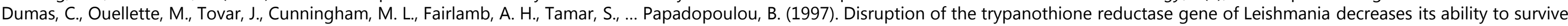
oxidative stress in macrophages. The EMBO Journal, 16(10), 2590-2598. https://doi.org/10.1093/emboj/16.10.2590

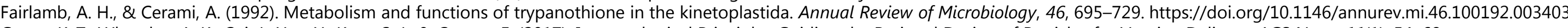

Gause, K. T., Wheatley, A. K., Cui, J., Yan, Y., Kent, S. J., \& Caruso, F. (2017). Immunological Principles Guiding the Rational Design of Particles for Vaccine Delivery. ACS Nano, 11(1), 54-68. https://doi.org/10.1021/acsnano.6b07343

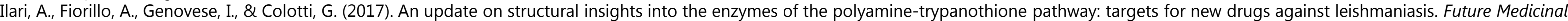
Chemistry, 9(1), 61-77. https://doi.org/10.4155/fmc-2016-0180

Irvine, D. J., Swartz, M. A., \& Szeto, G. L. (2013). Engineering synthetic vaccines using cues from natural immunity. Nature Materials, 12(11), 978-990. https://doi.org/10.1038/nmat3775

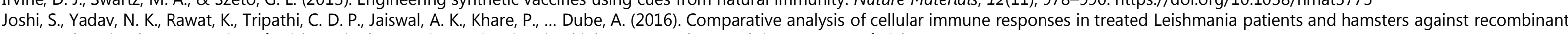
Th1 stimulatory proteins of Leishmania donovani. Frontiers in Microbiology, 7, 312. https://doi.org/10.3389/fmicb.2016.00312

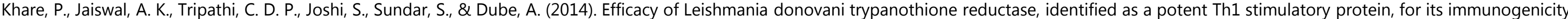
and prophylactic potential against experimental visceral leishmaniasis. Parasitology Research, 113, 851-862. https://doi.org/10.1007/s00436-013-3716-5

King, R. J., Campbell-Lendrum, D. H., \& Davies, C. R. (2004). Predicting Geographic Variation in Cutaneous Leishmaniasis, Colombia. Emerging Infectious Diseases, 10(4), 598-607. https://doi.org/10.3201/eid1004.030241

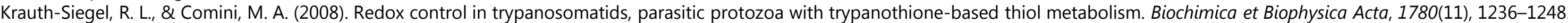
https://doi.org/10.1016/j.bbagen.2008.03.006 


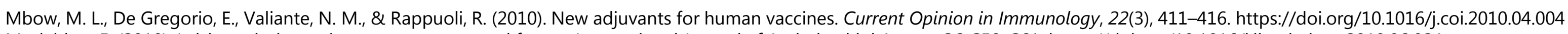

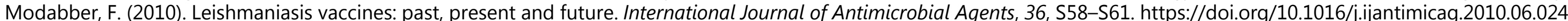

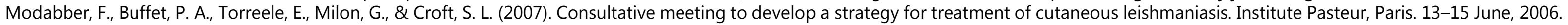
Kinetoplastid Biology and Disease, 6(1), 3. https://doi.org/10.1186/1475-9292-6-3

Monteiro, N., Martins, A., Reis, R. L., \& Neves, N. M. (2014). Liposomes in tissue engineering and regenerative medicine. Journal of The Royal Society Interface, 11(101), 20140459. https://doi.org/10.1098/rsif.2014.0459

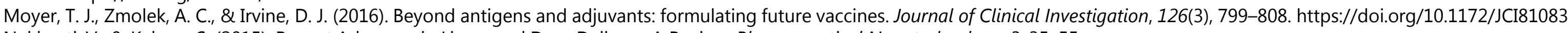
Nekkanti, V., \& Kalepu, S. (2015). Recent Advances in Liposomal Drug Delivery: A Review. Pharmaceutical Nanotechnology, 3, 35-55.

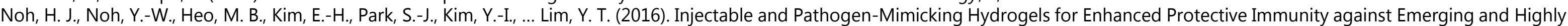
Pathogenic Influenza Virus. Small, 12(45), 6279-6288. https://doi.org/10.1002/smll.201602344

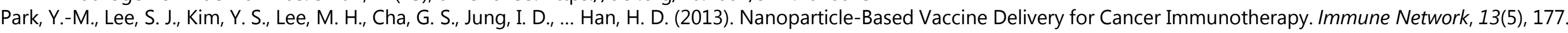
https://doi.org/10.4110/in.2013.13.5.177

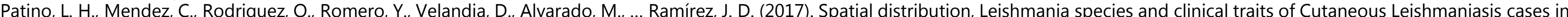
the Colombian army. PLOS Neglected Tropical Diseases, 11(8), e0005876. https://doi.org/10.1371/journal.pntd.0005876

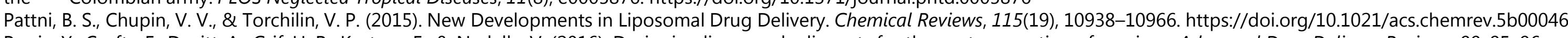

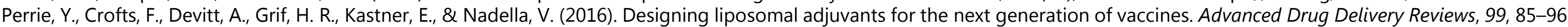
https://doi.org/10.1016/j.addr.2015.11.005

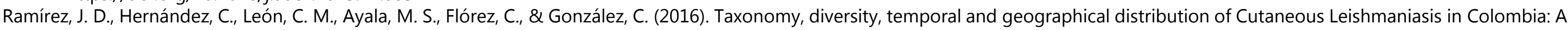
retrospective study. Scientific Reports, 6(1), 28266. https://doi.org/10.1038/srep28266

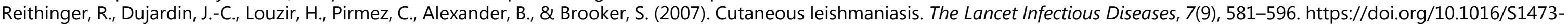
3099(07)70209-8

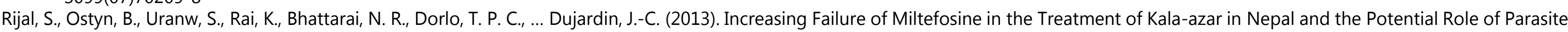
Drug Resistance, Reinfection, or Noncompliance. Clinical Infectious Diseases, 56(11), 1530-1538. https://doi.org/10.1093/cid/cit102

Skwarczynski, M., \& Toth, I. (2014). Recent advances in peptide-based subunit nanovaccines. Nanomedicine, 9(17), 2657-2669. https://doi.org/10.2217/nnm.14.187

Smith, D. M., Simon, J. K., \& Baker, J. R. (2013). Applications of nanotechnology for immunology. Nature Reviews Immunology, 13(8), 592-605. https://doi.org/10.1038/nri3488

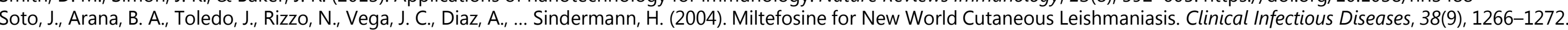
https://doi.org/10.1086/383321

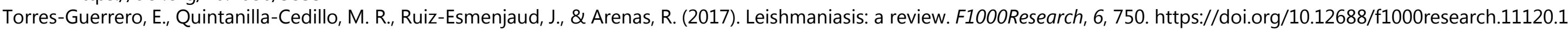

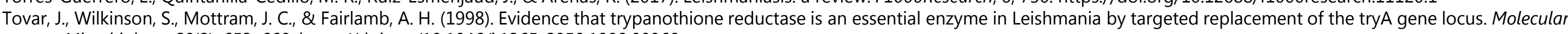
Microbiology, 29(2), 653-660. https://doi.org/10.1046/j.1365-2958.1998.00968.x

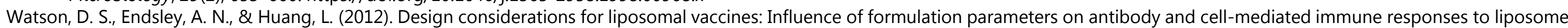
associated antigens. Vaccine, 30(13), 2256-2272. https://doi.org/10.1016/j.vaccine.2012.01.070

Yang, L., Li, W., Kirberger, M., Liao, W., \& Ren, J. (2016). Design of nanomaterial based systems for novel vaccine development. Biomaterials Science, 4(5), 785-802.

https://doi.org/10.1039/C5BM00507H

Zaman, M., Good, M. F., \& Toth, I. (2013). Nanovaccines and their mode of action. Methods, 60(3), 226-231. https://doi.org/10.1016/j.ymeth.2013.04.014 


\section{THANK YOU}

